\title{
THE HETEROGENEOUS MULTISCALE METHODS*
}

\author{
WEINAN $E^{\dagger}$ AND BJORN ENGQUIST $\ddagger$
}

\begin{abstract}
The heterogeneous multiscale method (HMM) is presented as a general methodology for the efficient numerical computation of problems with multiscales and multiphysics on multigrids. Both variational and dynamic problems are considered. The method relies on an efficient coupling between the macroscopic and microscopic models. In cases when the macroscopic model is not explicitly available or invalid, the microscopic solver is used to supply the necessary data for the macroscopic model. Scale separation can be exploited to considerably reduce the complexity of the microscopic solver. Besides unifying several existing multiscale methods such as the $a b$ initio molecular dynamics [13], quasicontinuum methods [73, 69, 68] and projective methods for systems with multiscales [34, 35], HMM also provides a methodology for designing new methods for a large variety of multiscale problems. A framework is presented for the analysis of the stability and accuracy of HMM. Applications to problems such as homogenization, molecular dynamics, kinetic models and interfacial dynamics are discussed.
\end{abstract}

\section{Introduction}

Many problems in nature involve multiple active scales. For example, chemical reactions may take seconds or hours, while the vibration of chemical bonds occurs at the time scale of femtoseconds $\left(10^{-15} s\right)$. The time scales for the internal motion of proteins and nucleic acids typically span from $10^{-14}$ seconds to seconds. Vortical structures in the atmosphere may range from meters to thousands of kilometers. If we want to understand in a reasonable detail the dynamics of dislocations that result in plastic deformation of a material, we face the task of studying the structure of dislocation cores at the scale of angstroms, to the collective dynamics of entangled dislocation loops at the scale of the size of the whole material [60].

With few exceptions, the traditional approach for such problems is to obtain either analytically or empirically explicit equations for the scale of interest, eliminating other scales in the problem. These equations are the basis for computer simulations. Indeed an impressive array of techniques have been developed to accomplish this task, including averaging methods in classical mechanics [4], homogenization theory [8], equilibrium statistical mechanics [47], WKB methods, nonequilibrium thermodynamics [20], kinetic theory [47], transition state theory [42], turbulence models, etc. This approach has been both successful, in fact it has so far dominated much of science and engineering, and necessary because of our restricted computational capabilities. A typical example of this approach is the Navier-Stokes equation in fluid mechanics, in which the microscopic processes are represented by an equation of state and the linear constitutive equations. It is quite impressive that general macroscopic conservation laws combined with empirical linear constitutive relations describe so well the dynamics of simple liquids in almost all situations.

Despite all its success, this approach also forces us to introduce empirical closures for other systems that are not always justified or understood. As a result, the success of such phenomenological equations is much less spectacular for a large class of complex systems. Typical examples of such a situation are found in complex fluids, plasticity, fracture dynamics, and important regimes of turbulent flows.

\footnotetext{
*Received: April 15, 2002; Accepted (in revised version): August 26, 2002.

$\dagger$ Department of Mathematics and PACM, Princeton University, USA, and School of Mathematics, Peking University, China.

$\ddagger$ Department of Mathematics and PACM, Princeton University,USA, and Department of Mathematics, University of California, Los Angeles,USA.
} 
A new approach, the "first principle" based approach, has emerged in recent years and has quickly attracted a great deal of attention. The aim of this approach is to model the theoretical input to a coarse-grained model from a more detailed microscopic model, bypassing the necessity of empirical modeling. Examples of such an approach include the $a b$ initio molecular dynamics [13] in which empirical potentials in molecular dynamics are replaced by "on the fly" electronic structure calculations, quasicontinuum method in which the stored-energy functional in nonlinear elasticity theory is replaced by molecular potentials [73], and coupled kinetic-hydrodynamic models of complex fluids in which constitutive relations are replaced by kinetic models for the conformation of macromolecules $[33,48,80]$. Such a coupled multiscale, multiphysics approach is discussed in many papers $[11,1,2,14,26,27,73,69,63,62,12,35,19]$ and is a central theme of the present paper. In a different direction, numerical methods that are based on partially resolving the relevant dynamical variables have been discussed thoroughly in $[15,16]$.

In what follows we will concentrate on two types of multiscale problems:

A. A macroscopic description is known but ceases to be valid in a localized region in space and/or time, and where the microscopic description has to be used instead. B. A macroscopic model may not be explicitly known or too expensive to obtain, but is known to exist; i.e., there exists a set of macroscopic variables obeying a closed macroscopic model.

Problems of Type A include defects in crystals where atomistic descriptions have to be used near the defects, and continuum theories are valid away from the defects [73]; contact line dynamics [21, 61]; turbulent flame fronts [59]; and chemical systems with localized chemical reactions where quantum mechanics has to be used in the chemically active regions, and classical mechanics can be used elsewhere. Problems of Type B are found in transport through inhomogeneous media such as porous medium flows and effective properties of composite materials [8], complex fluids, and plasticity. In principle there is also a class of problems, say of Type $\mathrm{C}$, which combines the characteristics of Types A and B, namely that the macroscopic model is not explicitly known and ceases to be valid near defects. This type of problem can be dealt with by combining the techniques for problems of Types A and B.

In this paper, we present a general framework for designing and analyzing numerical methods that deal with problems of Type A and Type B. The main motivation behind this framework is to make efficient usage of both the macroscopic and microscopic formulations even in cases when the macroscopic equations or models are not explicitly known. Our goal is to introduce a framework and analysis that cover many of the existing methods but also derives new techniques from the general formulation.

We will work with the following basic set-up. We have a microscopic process, such as molecular dynamics or quantum mechanics, that describes the microscopic state variable $u$ which is defined on a microscopic domain $\mathcal{D}$. We also have a macroscopic process that describes the macroscopic state variable $U$ which is defined on a macroscopic domain $D$. The two processes and state variables are related to each other by compression and reconstruction operators denoted by $Q$ and $R$ respectively: $Q u=U, R U=u$, with the property $Q R=I$ where $I$ is the identity operator. For example, if the microscopic process is described by kinetic theory, and the macroscopic process is described by hydrodynamics, then the compression operator maps the one-particle phase-space distribution function to the conserved mass, momentum, and energy densities. The reconstruction operator does the opposite and is, in general, not unique. Our interest is to resolve the macroscale behavior. In doing so, we 
sometimes have to make direct use of the microscale models. How that can be done efficiently is precisely the issue we address here.

Our aim is to accurately approximate the macroscopic state of the system. We do so by working with a macroscopic grid that resolves the large scale of the problem. There are two main components in the procedure. The first is to select a conventional macroscale scheme. The second is to estimate the necessary data for the macroscale scheme from the microscale model. The selection of the macroscale scheme depends on what we know about the macroscale model; e.g., whether it is a variational problem, whether it is conservative. When the macroscopic model is not fully known explicitly, we provide a general and efficient procedure for supplementing the missing data from microscopic models. For example, if the macroscopic problem is variational, and we use a standard finite element method, the task reduces to approximating the effective stiffness matrix for the macroscopic problem. This is done by solving the microscopic problem locally on a reduced computational domain. For dynamic problems, the missing data can be the macroscopic forces or fluxes or part of the forces or fluxes as the eddy viscosity terms in turbulence models. We estimate them using a generalization of the classical Godunov scheme for gas dynamics [38], namely at each macroscopic time step, we first reconstruct the microscopic state of the system from the known macroscopic state. We then evolve the microscopic process on a microscopic time scale until the desired macroscopic forces or fluxes reach a quasisteady value. We then use this estimated value to update the macroscopic state to the next macroscopic time step. In this way, we guarantee that the numerical results have a comparable accuracy to a full microscopic model. This procedure also sets up an approximation to the macroscopic model even though it is not explicitly used.

For problems of Type A, we simplify this algorithm by replacing the above procedure with a standard macroscopic solver in regions where the explicit macroscopic model is known and is known to be valid.

We call these methods the heterogeneous multiscale methods, abbreviated HMM, to emphasize the fact that different physical models and numerical techniques are used at different scales and different grids. In contrast, standard multigrid techniques are "homogeneous" in the sense that they employ the same physical model on different scales and are aimed at efficiently resolving the microscopic details $[12,40]$.

The key to the efficiency of such an approach is the possibility of reducing the temporal and state-space complexity of the microscopic model by exploiting scale separation. Such ideas can already be found in the literature; for example, for stiff ODEs in [39, 49, 5, 41, 34], and for kinetic schemes in gas dynamics in [79]. Our current work draws inspiration from the recent work of Kevrekidis and coworkers $[34,35]$. Closely related ideas are also found in [78].

This paper is written in a pedagogical style. In Section 2 we discuss the relations between macroscopic and microscopic models, the compression and reconstruction operators linking these models, and we give many examples. In Section 3 we discuss the overall scheme for HMM which contains two main ingredients: An overall macroscale scheme and the estimation of missing macroscale data from microscale models. In Section 4 we discuss how the microscopic models can be used efficiently to supplement the missing data for the macroscopic models. Stability and accuracy issues are discussed in Section 5. Algorithmic improvements are discussed in Section 6. Final conclusions are made in Section 7. Even though our emphasis is the overall framework, we also present new numerical techniques as well as some numerical results and analytical proofs. 


\section{The Macroscopic and Microscopic Models}

2.1. The Compression and Reconstruction Operators. It is convenient to think of the macroscopic and microscopic models as being defined on different domains with different state spaces linked by compression and reconstruction operators, as we discuss below.

Let $\{U\}$ be the set of macroscopic state variables that we are interested in, defined on a macroscopic computational domain (MCD) $D$. Denote by $\{u\}$ the set of microscopic state variables defined on the microscopic computational domain (mcd) $\mathcal{D}$. It is convenient to think of $\mathcal{D}$ as being a fiber bundle over $D$. We will denote by $\mathcal{D}_{x}$ the fiber over $x \in D$. We note that to be precise, the notion of fiber bundle has to be extended in an obvious way in order to accommodate the generality of our framework. Examples of the fibers will be given below. We will denote by $\Omega$ and $\omega$ the appropriate function spaces for the macroscopic and microscopic states respectively.

The macroscopic and microscopic state variables are related to each other by the compression and reconstruction operators, denoted by $Q$ and $R$ respectively:

$$
Q u=U, \quad R U=u
$$

Of course $Q$ and $R$ should satisfy

$$
Q R U=U
$$

Even though $Q$ and $R$ may be nonlinear operators, in the following exposition we will treat $Q$ as if it is linear. In general there is no unique way of defining $R$.

For the most part, we will concentrate our discussions on problems of Type B; i.e., problems for which there is a well-defined macroscopic model even though it may not be known explicitly. In the following, we will discuss how the macroscopic models are related to the microscopic models.

2.2. Variational Problems. Consider a microscopic minimization problem:

$$
\min _{u \in \omega} e(u) \text {. }
$$

Let $Q$ be an appropriately chosen compression operator:

$$
\min _{u} e(u)=\min _{U}\left(\min _{u: Q u=U} e(u)\right)
$$

If we define

$$
E(U)=\min _{u: Q u=U} e(u)
$$

on $\Omega$, then the macroscopic variational problem corresponding to $(2.3)$ is given by

$$
\min _{U \in \Omega} E(U)
$$

Let us consider a few examples.

Consider the variational problem

$$
\min _{u \in H_{0}^{1}(D)}\left\{\frac{1}{2} \int_{D} \sum_{i, j} a_{i, j}\left(x, \frac{x}{\varepsilon}\right) \frac{\partial u}{\partial x_{i}} \frac{\partial u}{\partial x_{j}} d x-\int_{D} f u d x\right\},
$$

where $a(x, y)$ is smooth and periodic in $y$ with period $I=[0,1]^{d}, f$ is smooth. There are two ways to look at this problem. The first is a numerical one. Consider a regular 
finite element triangulation of $D$, denoted by $T_{H}$, with mesh size $H$ that resolves the variations of $D$ and $f$, and the slow variables of $a$, but $H \gg \varepsilon$. Let $V_{H} \subset H_{0}^{1}(D)$ be the standard piecewise linear finite element space, and let $\Omega=V_{H}, \omega=H_{0}^{1}(D)$. For $u \in H_{0}^{1}(D)$ define $Q u=U \in V_{H}$, if

$$
\int_{D} \nabla u \cdot \nabla V d x=\int_{D} \nabla U \cdot \nabla V d x
$$

for all $V \in V_{H}$. It is easy to see that this uniquely defines $Q$. Now for $U \in \Omega=V_{H}$, let

$$
A^{\varepsilon}(U, U)=\min _{u \in H_{0}^{1}(\Omega), Q u=U} \int_{D} \sum_{i, j} a_{i, j}\left(x, \frac{x}{\varepsilon}\right) \frac{\partial u}{\partial x_{i}} \frac{\partial u}{\partial x_{j}} d x,
$$

then (2.6) is reduced to a problem on $\Omega=V_{H}$ :

$$
\min _{U \in V_{H}}\left\{\frac{1}{2} A^{\varepsilon}(U, U)-\int_{D} f U d x\right\} .
$$

The second way to think about this problem is to use homogenization theory. It is well known [8] that as $\varepsilon \rightarrow 0$, the solution $u^{\varepsilon}$ of (2.6) converges to the solution $U$ of the following problem:

$$
\min _{U \in H_{0}^{1}(D)}\left\{\frac{1}{2} \int_{D} \sum_{i, j} A_{i, j}(x) \frac{\partial U}{\partial x_{i}} \frac{\partial U}{\partial x_{j}} d x-\int_{D} f U d x\right\}
$$

where

$$
A_{i, j}(x)=\int_{I} \sum_{k, l} a_{k, l}(x, y)\left(\delta_{k i}-\frac{\partial \chi_{i}}{\partial y_{k}}\right)\left(\delta_{l j}-\frac{\partial \chi_{j}}{\partial y_{l}}\right) d y
$$

Here, for each fixed $x, \chi_{j}(x, y)(j=1, \ldots, d)$ is the solution of the cell problem

$$
\nabla_{y} \cdot\left(a(x, y)\left(\nabla_{y} \chi_{j}+e_{j}\right)\right)=0
$$

with periodic boundary condition on $I$ and $e_{j}=(0, \ldots 1, \ldots 0)$.

Now we can view (2.10) as our macroscopic problem with state space $\Omega=H_{0}^{1}(D)$. The macroscopic domain is $D$. The connection to the microscopic problem is via (2.11) where the microscopic state space $\omega=H_{0}^{1}(D \times I)$, and the microscopic domain is $\mathcal{D}=D \times I$ with $\mathcal{D}_{x}=I$, for $x \in D$.

It is important to note that macroscopic variational problems may also result from other types of microscopic models such as the ensembles averages in statistical physics:

$$
\langle g\rangle=\frac{\int_{\omega} g(u) e^{-\beta h(u)} d u}{\int_{\omega} e^{-\beta h(u)} d u} .
$$

This is an important line of research that we will pursue in the future.

2.3. Dynamic Problems. We will assume that the macroscopic model gives rise to a well-defined evolutionary process characterized by the solution operators $\{S(t)\}$ which form a semigroup: $U(t)=S(t) U(0)$, where $t \in I$, and $I$ is an index set, $I=$ an interval for continuous processes, and $I=\Delta t \mathcal{Z}$ for discrete processes. Here $\mathcal{Z}$ is the set of integers. The semigroup might be generated by a differential equation

$$
U_{t}=F(U),
$$


where $F(U)$ is in general a nonlinear operator on $U$, or a discrete evolutionary equation

$$
U^{n+1}=U^{n}+\Delta t F\left(U^{n}\right)
$$

or a set of transition rules

$$
\operatorname{Prob}\left(U \rightarrow U^{\prime}\right)=P\left(U, U^{\prime}\right)
$$

as in a kinetic Monte Carlo scheme. Likewise, the microscopic model is also assumed to give rise to a semigroup of solution operators denoted by $\{s(t)\}$, which might be generated by a differential equation

$$
u_{t}=f(u)
$$

where $f(u)$ is, in general, a nonlinear operator on $u$, or a discrete evolutionary equation

$$
u^{n+1}=u^{n}+\delta t f_{n}\left(u^{n}\right),
$$

or a set of transition rules

$$
\operatorname{Prob}\left(u \rightarrow u^{\prime}\right)=p\left(u, u^{\prime}\right) .
$$

We emphasize that the microscopic model is always assumed to be explicitly known.

Consider that there are two basic time scales of interest, a macroscopic time scale $t_{M}$ and a relaxation time scale for the microscopic process $t_{R}$. Assuming $t_{R}<<t_{M}$, our basic idea is to approximate $S(t)$ for $t_{R}<<t<t_{M}$ by

$$
S(t) U=Q s(t) R U
$$

for suitable reconstructions $R$. Knowing $S(t)$, we may find the effective macroscopic model through

$$
F(U)=\frac{1}{\Delta t}(S(\Delta t) U-U)
$$

for suitably chosen $\Delta t$ such that $t_{R}<<\Delta t<<t_{M}$. Next we give a few examples to illustrate these concepts.

2.3.1. Stiff ODEs. Consider the system

$$
\begin{aligned}
u_{t} & =f(u, v) \\
v_{t} & =\frac{1}{\varepsilon}(g(u)-v)
\end{aligned}
$$

where $\varepsilon$ is small. In this case the macroscopic (slow) variable is $u: U=u, Q(u, v)=$ $u=U$. The macroscopic equation is explicitly known in this case: $U_{t}=f(U, g(U))$.

In the general case when the slow and fast variables are not explicitly separated, we then have a slow manifold $\Omega$ in the state space $\omega$. In this case, the macroscopic equation may not be explicitly known.

2.3.2. Kinetic equations. We will use the classical Boltzmann equation as an example for illustration. But the ideas apply equally well to other kinetic models.

Consider the Boltzmann equation

$$
\frac{\partial f}{\partial t}+\left(\mathbf{v} \cdot \nabla_{\mathbf{x}}\right) f=\frac{1}{\varepsilon} B(f, f)
$$

where $f=f(\mathbf{x}, \mathbf{v}, t)$ is the phase-space one-particle distribution functions and $\varepsilon$ is the 
Knudsen number which is proportional to the mean free path between collisions. The collision operator at the right-hand side of (2.21) describes binary collisions between particles. The detailed form of $B$ is not important. What is crucial are the following properties satisfied by $B$ :

$$
\int_{R^{3}} B(f, f)(\mathbf{v}) \varphi(\mathbf{v}) d v=0, \varphi(\mathbf{v})=1, \mathbf{v},|\mathbf{v}|^{2}
$$

and

$$
\int_{R^{3}} B(f, f)(\mathbf{v}) \log f(\mathbf{v}) d v \leq 0 .
$$

Consequently, (2.21) admits a five-parameter family of equilibrium states,

$$
M(\rho, \mathbf{u}, T)(\mathbf{v})=\frac{\rho}{(2 \pi T)^{3 / 2}} \exp \left(-\frac{|\mathbf{v}-\mathbf{u}|^{2}}{2 T}\right),
$$

where $\rho, \mathbf{u}, T$ are the density, mean velocity, and temperature of the system, given by

$$
\rho=\int_{R^{3}} f(\mathbf{v}) d v, \mathbf{u}=\frac{1}{\rho} \int_{R^{3}} f(\mathbf{v}) \mathbf{v} d v, T=\frac{1}{3 \rho} \int_{R^{3}} f(\mathbf{v})|\mathbf{v}-\mathbf{u}|^{2} d v .
$$

In the hydrodynamic limit as $\varepsilon \rightarrow 0$, one obtains the following compressible Euler's equation for the conserved densities:

$$
\begin{gathered}
\frac{\partial \rho}{\partial t}+\nabla \cdot(\rho \mathbf{u})=0 \\
\frac{\partial}{\partial t}(\rho \mathbf{u})+\nabla \cdot(\rho \mathbf{u} \otimes \mathbf{u})+\nabla p=0 \\
\frac{\partial}{\partial t} E+\nabla \cdot((E+p) \mathbf{u})=0
\end{gathered}
$$

where $p=\rho T, E=\frac{1}{2} \rho|\mathbf{u}|^{2}+\frac{3}{2} \rho T$.

In this example, $(\rho, \mathbf{u}, T)$ are the macroscopic variables that we are interested in, and $f$ is the microscopic state variable. $Q f=(\rho, \mathbf{u}, T)$. The macroscopic domain is the physical domain, denoted by $D$, and the microscopic domain is $\mathcal{D}=D \times R^{3}, \mathcal{D}_{\mathbf{x}}=$ $\{\mathbf{x}\} \times R^{3}$, the momentum space.

2.3.3. Diffusion via random walks. Consider an ensemble of independent random walkers on a domain $D$. Let $U$ be the ensemble-averaged density of the random walkers; $\{u(t, y, w)\}$ be the position of the random walkers where $y \in D$ is the initial position of the random walkers; and $w \in \tilde{\Omega}$, the probability space of ensembles. $U=Q u=\langle u\rangle=\int_{\tilde{\Omega}} u(t, y, w) d P(w)$. For this problem $\mathcal{D}=D \times \tilde{\Omega}, \mathcal{D}_{x}=\tilde{\Omega}$.

2.3.4. Langevin equations and the kinetic Monte Carlo method. Consider a system of stochastic differential equations

$$
\frac{d x_{j}}{d t}=-\frac{\partial}{\partial x_{j}} V+\varepsilon \dot{B}_{j}(t), \quad j=1, \ldots, N
$$

where $V\left(x_{1}, \ldots, x_{N}\right)$ is the potential energy associated with the system and the $\dot{B}_{j}(t) \mathrm{s}$ are independent Gaussian white noise. For small $\varepsilon$, the system spends most of its time near the local minima of $V$, with rare and sudden transitions between different local 
minima. In such a situation, one can effectively approximate the dynamics of the full system by a Markov process whose state space is the set of local minima of $V$. The transition rates between different local minima can be obtained from the microscopic process (2.27) using the transition state theory [42]. An efficient numerical method for implementing this is discussed in [29].

In this example, the microscopic process is a set of ODEs and the macroscopic process is a discrete Markov process. For $x \in R^{N}, Q x=x^{*}$ if $x$ is in the domain of attraction of the local minimum $x^{*}$.

2.3.5. Molecular dynamics for fluids and solids. Consider a system of identical particles interacting via a short-ranged potential $U$. Their dynamics are described by Newton's law:

$$
\begin{aligned}
\frac{d}{d t} \mathbf{q}_{j}(t) & =\mathbf{p}_{j}(t) \\
\frac{d}{d t} \mathbf{p}_{j}(t) & =-\sum_{i \neq j} \nabla U\left(\mathbf{q}_{j}(t)-\mathbf{q}_{i}(t)\right)
\end{aligned}
$$

$j=1, \cdots N$. The corresponding Hamiltonian is

$$
H=\sum_{j=1}^{N} \frac{1}{2} \mathbf{p}_{j}^{2}+\frac{1}{2} \sum_{i \neq j} U\left(\mathbf{q}_{i}-\mathbf{q}_{j}\right) .
$$

Such a model is now used to simulate a very wide range of problems, from protein folding pathways, to crystal growth, defect dynamics in solids, complex fluids, etc. We will discuss the situation when molecular dynamics is used to model the macroscopic behavior of a system. We will first discuss the example of fluid flow.

In continuum theory, the state of a fluid is described by its density, velocity, and temperature fields, denoted by $\rho, \mathbf{u}$, and $T$ respectively. In most situations, neglecting dissipative effects, $(\rho, \mathbf{u}, T)$ obey the compressible Euler's equation (2.26), where $p=p(\rho, T)$ is the pressure, $e=e(\rho, T)$ is the internal energy density, and $E=\frac{1}{2} \rho \mathbf{u}^{2}+e$ is the total energy density.

The microscopic and macroscopic state variables are related in the following way. Given the microscopic state of the system $\left\{\mathbf{q}_{j}(t), \mathbf{p}_{j}(t)\right\}_{j=1, \cdots, N}$, we define the following empirical distributions:

$$
\begin{aligned}
\tilde{\rho}(\mathbf{x}, t) & =\sum_{j} \delta\left(\mathbf{q}_{j}(t)-\mathbf{x}\right) \\
\tilde{\mathbf{m}}(\mathbf{x}, t) & =\sum_{j} \mathbf{p}_{j}(t) \delta\left(\mathbf{q}_{j}(t)-\mathbf{x}\right) \\
\tilde{E}(\mathbf{x}, t) & =\sum_{j}\left\{\frac{1}{2} \mathbf{p}_{j}(t)^{2}+\frac{1}{2} \sum_{i \neq j} U\left(\mathbf{q}_{i}(t)-\mathbf{q}_{j}(t)\right)\right\} \delta\left(\mathbf{q}_{j}(t)-\mathbf{x}\right) .
\end{aligned}
$$

$(\tilde{\rho}, \tilde{\mathbf{m}}, \tilde{E})$ are the empirical density, momentum, and total energy fields from the microscopic model. They obey the equations 


$$
\begin{gathered}
\frac{\partial}{\partial t} \tilde{\rho}+\nabla \cdot \mathbf{j}_{\rho}(\mathbf{x}, t)=0, \\
\frac{\partial}{\partial t} \tilde{\mathbf{m}}+\nabla \cdot \tau(\mathbf{x}, t)=0, \\
\frac{\partial}{\partial t} \tilde{E}+\nabla \cdot \mathbf{j}_{E}(\mathbf{x}, t)=0,
\end{gathered}
$$

where the current densities are given by

$$
\begin{gathered}
\mathbf{j}_{\rho}(\mathbf{x}, t)=\sum_{j} \mathbf{p}_{j}(t) \delta\left(\mathbf{q}_{j}(t)-\mathbf{x}\right) \\
\tau_{\alpha \beta}(\mathbf{x}, t)=\sum_{j} p_{j \alpha}(t) p_{j \beta}(t) \delta\left(\mathbf{q}_{j}(t)-\mathbf{x}\right) \\
+\frac{1}{2} \sum_{i, j} \mathbf{F}_{\alpha}\left(\mathbf{q}_{j}(t)-\mathbf{q}_{i}(t)\right)\left(\mathbf{q}_{j}(t)-\mathbf{q}_{i}(t)\right)_{\beta} \int^{1} \delta\left(\lambda \mathbf{q}_{j}(t)+(1-\lambda) \mathbf{q}_{i}(t)-\mathbf{x}\right) d \lambda \\
\mathbf{j}_{E}(\mathbf{x}, t)=\sum_{j} \mathbf{p}_{j}(t)\left\{\frac{1}{2 m} \mathbf{p}_{j}(t)^{2}+\frac{1}{2} \sum_{i \neq j} U\left(\mathbf{q}_{j}(t)-\mathbf{q}_{i}(t)\right)\right\} \delta\left(\mathbf{q}_{j}(t)-\mathbf{x}\right) \\
+\frac{1}{2} \sum_{i \neq j} \frac{1}{2}\left[\left(\mathbf{p}_{j}(t)+\mathbf{p}_{i}(t)\right) \cdot \mathbf{F}\left(\mathbf{q}_{j}(t)-\mathbf{q}_{i}(t)\right)\right]\left(\mathbf{q}_{j}(t)-\mathbf{q}_{i}(t)\right) \\
\times \int_{0}^{1} \delta\left(\lambda \mathbf{q}_{j}(t)+(1-\lambda) \mathbf{q}_{i}(t)-\mathbf{x}\right) d \lambda
\end{gathered}
$$

where $\mathbf{F}=-\nabla U$. Ensemble or local time averages of the empirical conserved densities and currents give macroscopic fields and fluxes in the compressible Euler's equation.

For this problem $Q\left\{\mathbf{q}_{j}, \mathbf{p}_{j}\right\}=(\langle\tilde{\rho}\rangle,\langle\tilde{\mathbf{m}}\rangle,\langle\tilde{E}\rangle)$ where $(\tilde{\rho}, \tilde{\mathbf{m}}, \tilde{E})$ are defined in (2.29), and \langle\rangle denotes ensemble or spatial/temporal averaging. The fibers are either the probability space or the local spatial/temporal domains over which averaging is taken.

For solids it is customary to work with the Lagrangian coordinates. Let $\mathbf{y}(\mathbf{x}, t)$ be the deformed position of a material point whose undeformed position is $\mathbf{x}$, and $T_{0}$ be the Piola-Kirchoff stress tensor, and let $\rho_{0}(\mathbf{x})$ be the density of the material before deformation, and $\mathbf{q}_{0}$ be the heat flux. The macroscopic state variables are the displacement field $\mathbf{y}$, and the total energy density $E=\frac{1}{2} \rho_{0}|\dot{\mathbf{y}}|^{2}+e$ where $e$ is the internal energy density. The macroscopic equations take the form

$$
\begin{aligned}
\rho_{0} \ddot{\mathbf{y}} & =\nabla \cdot T_{0} \\
\dot{E} & =\nabla \cdot\left(T_{0} \dot{\mathbf{y}}\right)-\nabla \cdot \mathbf{q}_{0}
\end{aligned}
$$

For simplicity of notations, we will work with the one-dimensional situation. Generalization to higher dimensions is straightforward. Taking an arbitrary interval of macroscopic size $(A, B]$, we have

$$
\frac{d}{d t} \sum_{A<x_{j} \leq B} \dot{q}_{j}(t)=-\sum_{A<x_{j} \leq B} \sum_{k} \nabla U\left(q_{j}-q_{k}\right) .
$$


Here we have used $x_{j}$ to denote the initial position of the $j$-th particle. Notice that there are some cancellations in the last expression, which actually is equal to

$$
-\sum_{\substack{A<x_{j} \leq B \\ x_{k} \bar{\epsilon}(A, B]}} \nabla U\left(q_{j}-q_{k}\right) .
$$

Define the empirical stress $\tilde{T}_{0}$ by

$$
\tilde{T}_{0}(x, t)=\sum_{\substack{x_{j}<x \\ x_{k} \geq x}} \nabla U\left(q_{j}-q_{k}\right)
$$

we then have

$$
\frac{d}{d t} \sum_{A<x_{j} \leq B} \dot{q}_{j}(t)=-\left(\tilde{T}_{0}(B, t)-\tilde{T}_{0}(A, t)\right) .
$$

In the same fashion, let

$$
E_{A, B}=\sum_{A<x_{j} \leq B} \frac{1}{2} p_{j}(t)^{2}+\frac{1}{2} \sum_{A<x_{j} \leq B, k} U\left(q_{j}(t)-q_{k}(t)\right) .
$$

We then have

$$
\begin{aligned}
\frac{d}{d t} E_{A, B} & =-\frac{1}{2} \sum_{\substack{A<x_{j} \leq B \\
x_{k} \bar{\epsilon}(A, B]}}\left(\dot{q}_{j}+\dot{q}_{k}\right) \nabla U\left(q_{j}-q_{k}\right) \\
& =-\left(\tilde{J}_{E}(B, t)-\tilde{J}_{E}(A, t)\right)
\end{aligned}
$$

where

$$
\tilde{J}_{E}(x, t)=\frac{1}{2} \sum_{\substack{x_{j}<x \\ x_{k} \geq x}}\left(\dot{q}_{j}+\dot{q}_{k}\right) \nabla U\left(q_{j}-q_{k}\right) .
$$

Let $q(x, t)$ be the position of the particle whose initial position is nearest to $x$. We can write $\tilde{J}_{E}$ as

$$
\begin{aligned}
\tilde{J}_{E}(x, t) & =\dot{q}(x, t) \tilde{T}_{0}(x, t)+\frac{1}{2} \sum_{\substack{x_{j}<x \\
x_{k} \geq x}}\left(\dot{q}_{j}+\dot{q}_{k}-2 \dot{q}(x, t)\right) \nabla U\left(q_{j}-q_{k}\right) \\
& =\dot{q}(x, t) \tilde{T}_{0}(x, t)-\tilde{q}(x, t) .
\end{aligned}
$$

The last equation defines $\tilde{q}$ which is the discrete analog of heat flux.

Define the empirical distributions $\tilde{Y}, \tilde{E}$ through

$$
\begin{aligned}
& \int_{A}^{B} \tilde{Y}(x, t) d x=\sum_{A<x_{j} \leq B} q_{j}(t) \\
& \int_{A}^{B} \tilde{E}(x, t) d x=\sum_{A<x_{j} \leq B} \frac{1}{2} p_{j}(t)^{2}+\frac{1}{2} \sum_{A<x_{j} \leq B, k} U\left(q_{j}(t)-q_{k}(t)\right)
\end{aligned}
$$


Then we have

$$
\begin{aligned}
& \tilde{Y}_{t t}+\nabla \cdot \tilde{T}_{0}=0 \\
& \tilde{E}_{t}+\nabla \cdot \tilde{J}_{E}=0
\end{aligned}
$$

where $\tilde{T}_{0}$ and $\tilde{J}_{E}$ are the microscopic currents defined above. The macroscopic conserved densities and fluxes are related to the microscopic densities and currents through ensemble or spatial/temporal averaging. $Q\left\{q_{j}, p_{j}\right\}=(\langle\tilde{Y}\rangle,\langle\tilde{E}\rangle)$.

2.3.6. Interfacial dynamics. For simplicity, we will concentrate on the Ginzburg-Landau equation

$$
u_{t}^{\varepsilon}=\Delta u^{\varepsilon}+\frac{1}{\varepsilon^{2}} u^{\varepsilon}\left(1-\left(u^{\varepsilon}\right)^{2}\right)
$$

with $\varepsilon \ll 1$. One can think of this as a reaction-diffusion equation with fast reaction. Solutions to (2.32) are closely approximated by the constant equilibrium values $u=$ \pm 1 , except at the interfacial region of thickness $O(\varepsilon)$ where transition between the two equilibrium values takes place. The small scale in this problem corresponds to the internal structure of the interfacial region. The large scale corresponds to the overall shape of the interface.

The fast process in this problem is the reaction term, which vanishes at three points $u=-1,0,1$. These three points define the slow manifold: Given a function $u(x)$, the slow component of $u$ is the level set of $u$ at values $u=-1,1$, and 0 . Of particular interest is the level set $\{u=0\}$. Therefore given $u$, we define

$$
Q u=\left\{\begin{aligned}
1 & \text { if } u>0 \\
0 & \text { if } u=0 \\
-1 & \text { if } u<0
\end{aligned}\right.
$$

The dynamics of the macroscopic component of $u^{\varepsilon}$ then reduces to the dynamics of its level set $\left\{u^{\varepsilon}=0\right\}$, denoted by $\Gamma$.

2.3.7. Carleman equations. Our next example is the Carleman equation, which is a simple kinetic model. Later on we will use this example to present variations and limitations of HMM and to illustrate the importance of different scales.

$$
\begin{aligned}
& \frac{\partial u}{\partial t}+\frac{\partial u}{\partial x}=v^{2}-u^{2} \\
& \frac{\partial v}{\partial t}-\frac{\partial v}{\partial x}=u^{2}-v^{2}
\end{aligned}
$$

The initial values are assumed to be oscillatory,

$$
\begin{aligned}
& u(x, 0)=a(x, x / \varepsilon) \\
& v(x, 0)=b(x, x / \varepsilon)
\end{aligned}
$$

with $a(x, y), b(x, y)$ smooth and 1-periodic in $y$.

There exists a well-understood homogenization theory for this problem by Tartar, [74]. 


$$
\begin{aligned}
& \frac{\partial \tilde{u}}{\partial t}+\frac{\partial \tilde{u}}{\partial x}=\int_{0}^{1} \tilde{v}(x, y, t)^{2} d y-\tilde{u}^{2} \\
& \frac{\partial \tilde{v}}{\partial t}-\frac{\partial \tilde{v}}{\partial x}=\int_{0}^{1} \tilde{u}(x, y, t)^{2} d y-\tilde{v}^{2} \\
& \tilde{u}(x, y, 0)=a(x, y) \\
& \tilde{v}(x, y, 0)=b(x, y) .
\end{aligned}
$$

The two formulations both represent the microscale problem. In (2.34) the oscillations are given as a function of $x$ and $t$. In (2.35) the $\varepsilon$-scale is less explicit and only represented by its local distribution in the new independent variable $y$. Compare the discussion of fibers above. From [74] we have

$$
\lim _{\varepsilon \rightarrow 0}\left(\left|u(x, t)-\tilde{u}\left(x, \frac{x-t}{\varepsilon}, t\right)\right|+\left|v(x, t)-\tilde{v}\left(x, \frac{x+t}{\varepsilon}, t\right)\right|\right)=0 .
$$

The natural macroscale variables are the weak limits of the full microscale solutions and $Q$ is now defined as follows.

$$
\begin{aligned}
& U(x, t)=\int_{0}^{1} \tilde{u}(x, y, t) d y=\lim _{\delta \rightarrow 0}\left(\lim _{\varepsilon \rightarrow 0} \int K_{\delta}(x-z) u(z, t) d z\right) \\
& V(x, t)=\int_{0}^{1} \tilde{v}(x, y, t) d y=\lim _{\delta \rightarrow 0}\left(\lim _{\varepsilon \rightarrow 0} \int K_{\delta}(x-z) u(z, t) d z\right) .
\end{aligned}
$$

The kerned $K_{\delta}$ is compactly supported in $(-\delta, \delta)$ with

$$
\int_{-\delta}^{\delta} K_{\delta}(x) d x=1 .
$$

We will see in Section 5 that the reconstruction must contain some information of the microscale oscillations for HMM to be effective.

The relation between the macroscopic and microscopic models is illustrated in Figure 1. In this figure, the macroscopic model is defined in the $x$ space. The microstructures are defined on the $y$ space, the fibers. As we discussed above, the fibers can be the momentum space for the kinetic model, the periodic cell for the homogenization problems, the probability space of ensembles, etc.

\section{The Structure of HMM}

There are two main components in the heterogeneous multiscale method: $A n$ overall macroscopic scheme for $U$ and estimating the missing macroscopic data from the microscopic model.

3.1. The Overall Macroscopic Scheme. The right overall macroscopic sche- me depends on the nature of the problem, and typically there is more than one choice. For variational problems, we may use the standard finite element method. In fact, our examples in the next section use the standard piecewise linear finite element method. For dynamic problems that are conservative, we may use the methods developed for nonlinear conservation laws (see, e.g., [51]). Examples include the Godunov scheme, Lax-Friedrichs scheme, and the discontinuous Galerkin method. For dynamic problems that are nonconservative, we may simply use a standard ODE solver such 
as the forward Euler or the Runge-Kutta method, coupled with the force estimator that we discuss below.

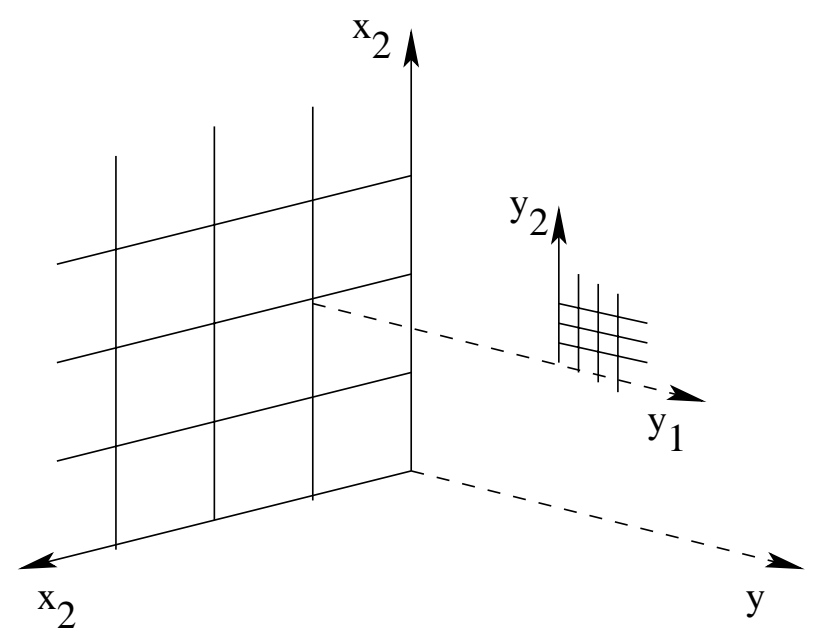

FIG. 1. Illustration of the macroscopic and microscopic computational domains. The $y$-spaces are the fibers representing the microstructures.

3.2. Estimation of the Macroscopic Data. After selecting the overall macroscopic scheme, we face the difficulty that not all data for the macro scheme are available since the underlying macro model is not explicitly known. The next component of HMM is to estimate such missing data from the microscopic model. This is done by solving the micro model locally, subject to the constraint that $\tilde{Q} u=U$ where $\tilde{Q}$ is the approximation to $Q$ and $U$ is the current macro state. For example, for the variational homogenization problem, the missing data is the stiffness matrix for the macro model. As we explain in the next section, this data can be estimated by solving the original microscopic variational homogenization problem on a unit cell in each element of the triangulation, subject to the constraint that $\tilde{Q} u=U$. For dynamic problems, such data can be estimated from a Godunov procedure, namely, that we first reconstruct the micro state from $U$, and evolve the micro state subject to the constraint that $\tilde{Q} u=U$, and then estimate the missing data from $u$. Such a reconstruction/micro evolution/data-processing procedure is very much analogous to the reconstruction/evolution/cell-averaging procedure in Godunov scheme. The missing data can be either the forces or fluxes or a part of the forces or fluxes such as the eddy viscosity term in a turbulence model. We also have the option of carrying out a number of such microscopic calculations (e.g. with different reconstruction or different realization of the randomness) and extract a more accurate estimate from the collection of microscopic calculations.

3.3. Examples. To illustrate the selection of the macroscale scheme and the estimation of missing macroscale data from microscale models, we will discuss some examples in more detail.

3.3.1. Variational problems. Examples include:

1.

$$
\min _{u \in H_{0}^{1}(D)} \int_{D}\left\{\frac{1}{2} \sum_{i, j} a_{i, j}^{\varepsilon}(x, u) \frac{\partial u}{\partial x_{i}} \frac{\partial u}{\partial x_{j}}-f(x) u(x)\right\} d x
$$


where the multiscale nature of the problem is contained in the tensor $a^{\varepsilon}(x, u)=$ $\left(a_{i, j}^{\varepsilon}(x, u)\right)$ which can be of the form

(a) $a^{\varepsilon}(x, u)=a\left(x, \frac{x}{\varepsilon}\right)$, where $a(x, y)$ is smooth and periodic in $y$ with period $[0,1]^{d}$. This is the classical homogenization problem we discussed earlier.

(b) $a^{\varepsilon}(x, u)=a\left(x, \frac{x}{\varepsilon}\right)$, where $a(x, y)$ is random and stationary in $y$. This can be used to model random medium.

(c) $a^{\varepsilon}(x, u)=a\left(x, u, \frac{x}{\varepsilon}\right)$, where $a(x, u, y)$ is smooth. The dependence on $u$ makes this problem nonlinear. The dependence on $y$ can be either periodic or random stationary.

The macroscale problem is of the type

$$
\min _{U \in H_{0}^{1}(D)} \int_{D}\left\{\frac{1}{2} A(x, U, \nabla U)-f(x) U(x)\right\} d x .
$$

2. Atomistic models of crystalline solids:

$$
\min _{\left\{x_{j}\right\}} \sum_{y_{i}, y_{j} \in D} V\left(x_{i}-x_{j}\right)
$$

subject to loading conditions, where $V$ is a pairwise atomistic potential, $x_{i}=y_{i}+u_{i}, y_{i}$ is the position of the $i$-th atom before deformation, $u_{i}$ is the displacement of the $i$-th atom. The macroscale problem is of the type considered in nonlinear elasticity

$$
\min _{U} \int_{D} f(\nabla U)
$$

where $U$ is the macroscale displacement field.

For these problems, we can choose the macroscale scheme to be the standard finite element method over a macroscale triangulation. The macroscale data that we need to estimate is either $\int_{D} A(x, U, \nabla U) d x$ or $\int_{D} f(\nabla U) d x$ for $U \in V_{H}$, the finite element space. These can be approximated via the following steps.

1. For each element $K$, approximate $\int_{K} A(x, U, \nabla U) d x$ or $\int_{K} f(\nabla U) d x$ by a quadrature formula.

2. For each quadrature nodes $x_{i} \in K$, approximate $A(x, U, \nabla U)\left(x_{i}\right)$ or $f(\nabla U)\left(x_{i}\right)$ by minimizing the original microscale problem over a microcell $\Delta_{x_{i}}$, subject to the constraint that $\int_{\Delta_{x_{i}}} u(x) d x=\int_{\Delta_{x_{i}}} U(x) d x, \int_{\Delta_{x_{i}}} \nabla u(x) d x=\int_{\Delta_{x_{i}}} \nabla U(x) d x$, with appropriate changes for the atomistic problem. For the periodic homogenization and crystalline solids problems, $\Delta_{x_{i}}$ can be chosen to be a unit cell around $x_{i}$, if we replace the constraint by a periodic boundary condition or the Cauchy-Born rule, as we explain in the next section. For the stochastic homogenization problem, $\Delta_{x_{i}}$ should be larger than the correlation length. In this case, it may also be advantageous to perform ensemble averages over several realizations of $a\left(x, \frac{x}{\varepsilon}\right)$.

3.3.2. Dynamic Problems of Conservative Type. Examples include 1 .

$$
\partial_{t} u^{\varepsilon}=\nabla \cdot\left(a^{\varepsilon}(x, u) \nabla u^{\varepsilon}\right)
$$

where $\left\{a^{\varepsilon}(x, u)\right\}$ is as discussed above.

2 .

$$
\partial_{t} u^{\varepsilon}+\nabla \cdot\left(a^{\varepsilon}(x) u\right)=0
$$

where $a^{\varepsilon}(x)=a\left(x, \frac{x}{\varepsilon}\right), a(x, y)$ can either be periodic or stochastic stationary in $y$. 
3. Kinetic models such as the Boltzmann or BGK equations.

4. Molecular dynamics of the type discussed in Section 2.

5. Spin-exchange models via Kawasaki dynamics [71].

Other examples may include models of phase segregation, mixtures of binary fluids, elastic effects, etc. The macroscale models are of the type

$$
U_{t}+\nabla \cdot J=0
$$

where $U$ is, in general, a vectorial macroscale variable, $J$ may depend on $x, U, \nabla U$, etc.

The macroscale scheme can be either a finite volume method such as the Godunov scheme or a finite element method such as the discontinuous Galerkin method. We will discuss here the finite volume method. HMM based on the discontinuous Galerkin method is considered in [25].

The missing macroscale data for a finite volume method for (3.1) is the macroscale flux $J$ at the cell boundaries, denoted by $\left\{J_{j+\frac{1}{2}}\right\}$. They can be estimated by the following "Godunov-like" procedure:

1. Select a microcell $\Delta_{j+\frac{1}{2}}$ around the cell boundary at $x_{j+\frac{1}{2}}$.

2. From $\left\{U_{j}^{n}\right\}$, reconstruct the microstates $\{\tilde{u}\}$ on $\left\{\Delta_{j+\frac{1}{2}}\right\}$. $\tilde{u}$ should be consistent with $\left\{U_{j}^{n}\right\}$ in the sense that $\tilde{Q} u=U^{n}$, where $\tilde{Q}$ is the approximation of $Q$ restricted to $\left\{\Delta_{j+\frac{1}{2}}\right\}$.

3. Evolve the microstate $u(t)$ using the microscale model inside $\left\{\Delta_{j+\frac{1}{2}}\right\}$, with initial state $\{\tilde{u}\}$, and subject to the constraint that

$$
\tilde{Q} u(t)=U \text {. }
$$

4. Evaluate the macroscale flux $\left\{J_{j+\frac{1}{2}}\right\}$ using $\{u(t)\}$.

The constraint $\tilde{Q} u=U$ requires some additional comment. Take the example of molecular dynamics. If we would like to capture the macroscale behavior at the level of Euler's equations, the constraint is simply that the average mass, momentum, and energy should be given by the prescribed macroscale values given by $\left\{U^{n}\right\}$. If we would like to capture the viscous or higher order effects, we also need to constrain the system such that the average density, momentum, and energy gradients be given by the macroscale values.

The rules for selecting $\left\{\Delta_{j+\frac{1}{2}}\right\}$ are the same as for the variational problems. As usual for periodic homogenization and crystalline solids problems, $\Delta_{j+\frac{1}{2}}$ can be chosen to be the unit cell.

3.3.3. Dynamic problems of nonconservative type. Examples include:

1 .

$$
\partial_{t} u^{\varepsilon}=\sum_{i, j} a_{i, j}^{\varepsilon}(x, u) \frac{\partial^{2} u}{\partial x_{i} \partial x_{j}}
$$

where $\left\{a^{\varepsilon}(x, u)\right\}$ is as discussed before.

2. Spin flip models [36] that lead to Ginzburg-Landau type of equations.

In this case, we write the macroscale model as

$$
U_{t}=F(U)
$$

where $F(U)$ can be a nonlinear operator acting on $U$. For the macroscale scheme, we choose an ODE solver on a grid, such as forward Euler or Runge-Kutta, and we need to estimate $F(U)$ on the macro grid. 
For each macro gridpoint $x_{j}$, we again select a microcell $\Delta_{j}$ around $x_{j}$. The principle for selecting $\Delta_{j}$ is the same as before. From $\left\{U_{j}^{n}\right\}$, we construct a piecewise polynomial of $k$-th order in $\Delta_{j}$ denoted by $U_{j}^{n}(x)$. The rest of the steps are the same as that for the conservative systems. We note that the constraint $\tilde{Q} u=U$ can be interpreted as

$$
\int_{\Delta_{j}}\left(u(x)-U_{j}^{n}(x)\right) x^{m} d x=0
$$

for $0 \leq m \leq k$.

3.3.4. Macroscale markov chains. When the macroscale process is a Markov chain, it is natural to use a kinetic Monte Carlo method as the macro scheme. The missing data might be the transition rates between macro states. Estimating such data is a rather nontrivial task. It is discussed in [29].

\section{Estimating the Macroscale Data}

In this section, we discuss in some detail the estimation of the missing macroscale data by solving local microscale problems. There are two main issues. For consistency and accuracy, the microscale problems have to be reformulated correctly by adding constraints such as $\tilde{Q} u=U$ or boundary conditions. For efficiency, we have to exploit possible reduction of the complexity of the microscale problems. This can be done by either reducing the size of the computational domains for the microscale problem through compression or a semi-empirical method as we explain later in this section.

4.1. Compressed Microscopic solvers. The key idea for the efficiency of the proposed method is that of compression; i.e., to reduce the computational complexity of the microscopic problem through a reduction of the size of the microscopic computational domain (mcd) by exploring the separation of the macroscopic and the microscopic scales. Other compression techniques are found in, e.g., [31].

4.1.1. Variational problems. Again it is best to illustrate the main ideas with a few examples. We will first discuss the classical homogenization problem discussed in Section 2.2.

For $u \in H^{1}(D)$, let

$$
a^{\varepsilon}(u, u)=\frac{1}{2} \int_{D} \sum_{i, j} a_{i, j}^{\varepsilon}(x) \frac{\partial u}{\partial x_{i}} \frac{\partial u}{\partial x_{j}} d x .
$$

Define the compression operator $Q$ as in Section 2.2, then

$$
A^{\varepsilon}(U, U)=\min _{u \in H_{0}^{1}(D), Q u=U} a^{\varepsilon}(u, u)
$$

for $U \in V_{H} . A^{\varepsilon}$ is often not explicitly known. It is needed in evaluating the stiffness matrix for the macroscale finite element method. For this problem, the approximate macroscopic solver reduces to an approximate evaluation of the stiffness matrix associated with $A^{\varepsilon}(U, U)$.

First we consider the case when $a^{\varepsilon}(x)=a\left(x, \frac{x}{\varepsilon}\right)$, where $a(x, y)$ is periodic in $y$ with period $I=[0,1]^{d}$. To obtain an approximation of $A^{\varepsilon}(U, U)$, we consider, on each triangle $K$, the solution of a "cell problem"

$$
\nabla \cdot\left(a\left(x, \frac{x}{\varepsilon}\right) \nabla u_{K}(x)\right)=0
$$


on $x_{K}+\varepsilon I$, where $x_{K}$ is an interior point of $K$, subject to the condition that

$$
u_{K}(x)-U(x) \text { is periodic. }
$$

Let

$$
\tilde{A}(U, U)=\sum_{K} \frac{|K|}{\varepsilon^{d}} \int_{x_{K}+\varepsilon I} \sum_{i, j} a_{i, j}\left(x, \frac{x}{\varepsilon}\right) \frac{\partial u_{K}}{\partial x_{i}} \frac{\partial u_{K}}{\partial x_{j}} d x
$$

where $|K|$ denotes the volume of $K$. We define this to be the approximation to $A^{\varepsilon}(U, U)$ needed to assemble the stiffness matrix for the macroscopic problem.

In the general case when $a^{\varepsilon}$ does not have the form $a\left(x, \frac{x}{\varepsilon}\right)$, we define $\tilde{A}_{K}$ by

$$
\tilde{A}_{K}(U, U)=\min _{u} \frac{1}{|\tilde{K}|} \int_{\tilde{K}} \sum_{i, j} a_{i j}^{\varepsilon}(x) \frac{\partial u}{\partial x_{i}} \frac{\partial u}{\partial x_{j}} d x
$$

subject to the condition $\int_{\tilde{K}} \nabla u d x=\int_{\tilde{K}} \nabla U d x$, where $\tilde{K}$ is some appropriately chosen subdomain of $K$. We then let

$$
\tilde{A}(U, U)=\sum_{K}|K| \tilde{A}_{K}(U, U)
$$

and use $\tilde{A}$ as an approximation to $A^{\varepsilon}$. A good example of such a situation is when $a(x, y)$ is random and stationary in $y$; in this case, the size of such approximate cell problems (4.6) has to be larger than the local correlation length of $a$.

This method is based on the strategy of solving directly the macroscopic problems and approximate the macroscopic stiffness matrices by solving "the microcell problems." The computational complexity of this method does not increase as $\varepsilon$ is decreased. Furthermore, as we show below, it extends easily to nonlinear and timedependent problems. In contrast, the method proposed in [7] was based on the idea of replacing the finite element basis functions with functions having the correct microstructures. This has the disadvantage that the basis functions are expensive to compute. It is particularly difficult to extend such methods to nonlinear problems, or problems for which the microstructure evolves in time. [45] extends the method of [7] to high dimensions. For other related work, see [46, 66, 67].

The quasicontinuum method can be formulated in the same way. Here our problem is to simulate the nonlinear elastic behavior of crystalline solids without using a stored-energy functional, and we base our simulation on the molecular potential $V\left(x_{1}, \ldots x_{N}\right)$ where the $\left\{x_{j}\right\}$ 's are the positions of the atoms. As before, we start with a macroscopic triangulation of the sample and denote by $V_{H}$ the standard (vectorial) piecewise linear finite element space. The microscopic state variable is now the positions of the atoms $u=\left\{x_{1}, \ldots x_{N}\right\}$. We say $Q u=U \in V_{H}$, if the average strain between the atoms on the triangle $K$ is equal to the average strain of $U$ on $K$ for each $K$. The reduced macroscopic energy functional $E(U)$ should be given by

$$
E(U)=\min _{u, Q u=U} e(u)
$$

where $e(u)$ is the total potential energy associated with the configuration $u$. The quasicontinuum method approximates $E(U)$ using the Cauchy-Born rule. For each triangle $K$, let $E_{K}(U)$ be the potential energy of a unit cell subject to the constant strain $\nabla U$ on $K$, and let

$$
\tilde{E}(U)=\sum_{K} n_{K} E_{K}(U)
$$


where $n_{K}$ is the number of unit cells in $K . \tilde{E}(U)$ is the quasicontinuum approximation of $E(U)[73,69,68]$.

The quasicontinuum method developed by Tadmor et al. has an additional important twist allowing it to deal with problems of Type C. In this case, quasicontinuum method provides the option of returning to a full-atom computation for $E(U)$ near defects where the local strain varies on an atomic scale.

In summary, the basic strategy for compressing the state space complexity of the microscale model is to reduce the computation on each macroscale cell to a basic microscopic unit. Such microscopic units are the single period in a periodic homo-genization problem, a unit cell in crystalline materials [73], or a block of size comparable to the correlation length of the medium in porous medium flows.

4.1.2. Dynamic problems. For concreteness, let us assume that the macroscopic model takes the form of a differential equation $U_{t}=F(U)$. Given $U$, we would like to estimate $F(U)$ from a microscopic model, say (2.17).

Our basic assumption is that the local relaxation time of the microscopic process is much shorter than typical time scales for the macroscopic process: $t_{R} \ll t_{M}$. Our starting point is the following generalization of the gas-kinetic scheme [79] for the approximation of $F(U)$ (see also [35]).

Given $U^{n}, F(U)$ is estimated via the following steps:

1. Reconstruction: Reconstruct the microscopic state $\tilde{u}$ from $U^{n}, \tilde{u}=R U^{n}$.

2. Microscopic evolution: Evolve the microscopic process on a reduced computational domain up to some appropriate time $\alpha \Delta t, u(t)=s(t) \tilde{u}, t^{n} \leq t \leq t^{n}+\alpha \Delta t$.

3. Force estimation: Obtain an approximation to $F(U)$ by compression of the microscopic forces. If the microscopic process has the form of a differential equation $u_{t}=f(u)$, then we can write

$$
F\left(U^{n}\right)=\tilde{Q}\left\{f(u(t)): t^{n} \leq t \leq t^{n}+\alpha \Delta t\right\}
$$

where $\tilde{Q}$ is a space-time compression operator.

4. Macroscopic evolution: Having an estimation of $F\left(U^{n}\right)$, we can then compute $U^{n+1}$ using standard ODE solvers.

(4.8) is called an $F$-estimator.

In practice, Step 3 should proceed simultaneously with Step 2. The value of $\alpha$ is determined by when the $F$-estimator reaches a nearly stationary value.

If the macroscopic process is a kinetic Monte Carlo type of Markov process, the force estimation is replaced by estimating the transition probabilities between macroscopic states. Such a $P$-estimator is discussed in [29].

It remains to discuss three specific details of the above algorithm.

1. Reconstruction.

2. Compression; i.e., reduction of the computational complexity of the microscopic solver by reducing the size of the computational domain.

3. Force estimation. The data from the microscopic solver has to be processed in order to provide an accurate estimation of the macroscopic forcing term.

These three issues are related. We will center our discussion on compression. We will discuss two aspects of compression: compression in space and compression in time.

The basic principle for compression in space is the same as for the variational problems; i.e., by reducing the computation on each macroscopic cell to a "microscopic 
unit." For the homogenization problems,

$$
\begin{gathered}
\partial_{t} u^{\varepsilon}=\nabla \cdot\left(a\left(x, \frac{x}{\varepsilon}\right) \nabla u^{\varepsilon}\right) \\
\partial_{t} u^{\varepsilon}+\nabla \cdot\left(a\left(x, \frac{x}{\varepsilon}\right) u^{\varepsilon}\right)=0
\end{gathered}
$$

this leads to the following algorithms for approximating the local averages of $u^{\varepsilon}$ on a macroscopic grid:

1. Reconstruction. From $\left\{U_{j}^{n}\right\}$, construct a piecewise polynomial $U^{n}(x)$. The order of the polynomial depends on what problem we solve. It should be at least piecewise quadratic for (4.9), and piecewise linear for (4.10). We will return to this issue in Section 5 when we discuss the accuracy of this method.

2. For each macroscopic cell $\Delta$, solve (4.9) or (4.10) on $x_{\Delta}+\varepsilon I$ subject to the boundary condition that $u(x, t)-U^{n}(x)$ is periodic with period $\varepsilon I$.

3. Let $F_{j}^{n}$ be the average on $x_{\Delta_{j}}+\varepsilon I$ of the right-hand side of (4.9) or (4.10).

4. Compute $\left\{U_{j}^{n+1}\right\}$ using standard ODE solvers and the $F$-estimator $\left\{F_{j}^{n}\right\}$.

The quasicontinuum method also has a natural extension in the same fashion to dynamic problems. The easiest setting for doing so is the finite element method. We would like to solve the elastic wave equation

$$
\frac{\partial^{2}(U(t), V)}{\partial t^{2}}=-\left(\frac{\partial E}{\partial U}(U(t)), V\right)
$$

where $U(t), V \in V_{\Delta}$ and the functional $E(U)$ is defined through molecular potentials, via the Cauchy-Born rule, in exactly the same way as was done in quasicontinuum method.

We next turn to compression in time. By resorting to the microscopic model in order to simulate the macroscopic dynamics, we are forced to resolve the microscopic time scales which are not of interest. This is particularly expensive if $t_{R} \ll t_{M}$. However, in this case we can explore this time scale separation to reduce the computational cost in the temporal domain.

Let us express the $F$-estimator in the form

$$
\tilde{F}(U)=Q \sum_{j=1}^{k} \psi_{j} f\left(u_{j}\right)
$$

where the weights $\left\{\psi_{j}\right\}$ should satisfy

$$
\sum_{j=1}^{k} \psi_{j}=1
$$

$u_{j}$ is the computed microscopic state at microscopic time step $j$. It is helpful to distinguish two different scenarios by which relaxation to local equilibrium takes place. For some problems, such as the parabolic homogenization problem (4.9) and the Boltzmann equation, we have strong convergence to equilibrium. No temporal or ensemble averaging is necessary for the convergence of the physical observables. For other problems, such as the advection homogenization problem and molecular dynamics, convergence to equilibrium is in the sense of distributions; i.e., physical observables converge to their local equilibrium values after time or ensemble averaging. The selection of the weights in the $F$-estimator crucially depends on the nature of this 
convergence. In particular, we note two special choices. The first is: $\psi_{k}=1$ and $\psi_{j}=0$ for $j<k$. This is suitable when we have strong convergence to equilibrium. The second choice is: $\psi_{j}=\frac{1}{k}$, for $1 \leq j \leq k$. This is more suited for the case when we have weak convergence to local equilibrium. More accurate choices of the weights are discussed in [23].

The time interval on which the microscopic model has to be solved depends on how fast the transient introduced by the reconstruction step dies out. This is best illustrated by examples. Consider the parabolic homogenization problem

$$
u_{t}^{\varepsilon}=\nabla \cdot\left(a\left(x, \frac{x}{\varepsilon}\right) \nabla u^{\varepsilon}\right)
$$

on $D$, with Dirichlet boundary condition $\left.u^{\varepsilon}\right|_{\partial D}=0$. To approximate the macroscopic behavior of $u^{\varepsilon}$, we will work with a macroscopic grid of size $(\Delta x, \Delta t)$. Let $U=Q u^{\varepsilon}$ be the moving cell averages of $u^{\varepsilon}$ over a cell of size $\Delta x$. Let $R$ be the piecewise linear reconstruction. In one dimension, this is $R U(x)=U_{j}+\frac{U_{j+1}-U_{j}}{\Delta x}\left(x-x_{j}\right)$, for $x \in[j \Delta x,(j+1) \Delta x]$. With this reconstruction, we proceed with the microscopic solver. Asymptotic analysis suggests that the relaxation time for this problem is $O\left(\varepsilon^{2}\right)$ [8]. We plot in Figure 2 a typical behavior of the microscopic flux $j^{\varepsilon}(x, t)=$ $a\left(x, \frac{x}{\varepsilon}\right) \nabla u^{\varepsilon}(x, t)$ at a cell boundary over the time interval $\left[t^{n}, t^{n}+\Delta t\right]$ as a function of the micro time steps. It is quite clear that $j^{\varepsilon}(x, t)$ quickly settles down (after about 35 micro time steps) to a quasistationary value after a rapid transient. We obtain an efficient numerical scheme if we select this value as the macroscopic flux and use that to evolve $U$ over a much larger time step $\Delta t$.
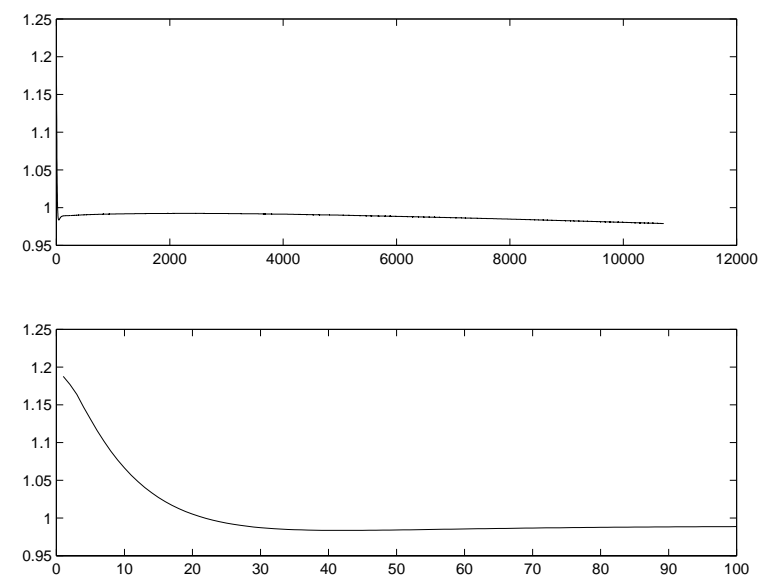

FIG. 2. Computed flux $\tau^{\varepsilon}(x, t)=a\left(x, \frac{x}{\varepsilon}\right) \nabla u^{\varepsilon}(x, t)$ as a function of the micro time step over one typical macro time step, for the parabolic homogenization problem with a $\left(x, \frac{x}{\varepsilon}\right)=$ $2+\sin 2 \pi \frac{x}{\varepsilon}$. The bottom figure is a detailed view of the top figure for small time steps. Notice that $j^{\varepsilon}(x, t)$ quickly settles down (after about 35 micro time steps) to a quasistationary value after a rapid transient.

Our next example is the advection homogenization problem

$$
u_{t}^{\varepsilon}+\nabla \cdot\left(a\left(x, \frac{x}{\varepsilon}\right) u^{\varepsilon}\right)=0
$$

in one dimension. We assume $a(x, y)>a_{0}>0$. We proceed as before except that we take a piecewise constant reconstruction. In contrast to the previous example, the 
temporal oscillations in the solutions of (4.12) do not die out. This is reflected in Figure 3 where we plot the microscopic flux $j^{\varepsilon}(x, t)=a\left(x, \frac{x}{\varepsilon}\right) u^{\varepsilon}(x, t)$ over the time interval $\left[t^{n}, t^{n}+\Delta t\right]$ as a function of the microscale time steps. $j^{\varepsilon}$ remains oscillatory throughout the time interval. Nevertheless, if we plot the time average

$$
\bar{j}(x, t)=\frac{1}{t} \int_{t^{n}}^{t^{n}+t} K\left(1-\frac{\tau}{t}\right) j^{\varepsilon}(x, \tau) d \tau, \quad K(\tau)=1-\cos 2 \pi \tau
$$

as shown in Figure 3, we see that it settles down to a quasistationary value on a time scale of $O(\varepsilon)$.

The fact that the microscopic process only has to be evolved on time scales comparable to $t_{R}$ leads to other possibilities of state space compression by neglecting the part of the state space which does not contribute significantly to the $F$-estimator.

Truncation of the mcd introduces artificial numerical boundaries where boundary conditions have to be imposed. We suggest either periodic or free boundary conditions subject to the constraint that $\tilde{Q} u=U$. Ideally one should apply the absorbing boundary conditions that aim at eliminating the spurious effect of the artificial boundary. Such boundary conditions were proposed in the context of wave equations in [18, 32], and for molecular dynamics in [26, 27]. However, extending them to general situations does not seem to be a simple task, and we will take it up in future work.
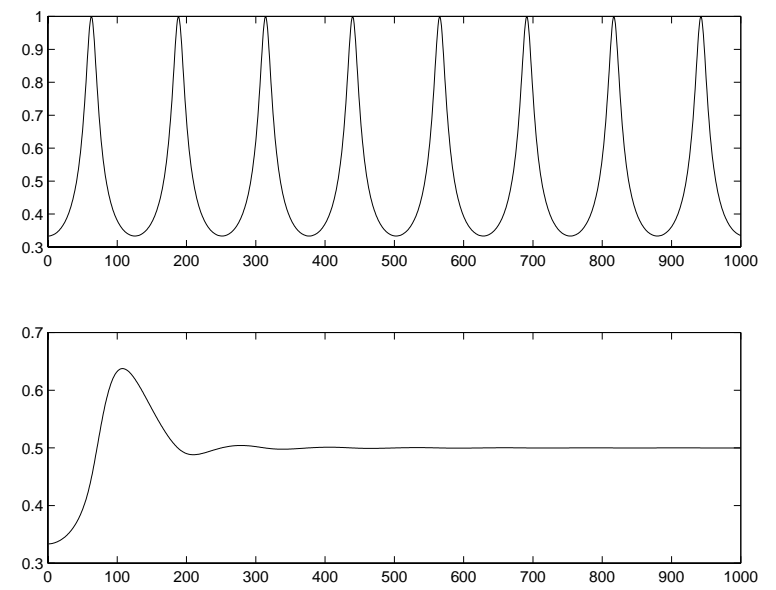

FIG. 3. Top figure: Computed flux $j^{\varepsilon}(x, t)=a\left(x, \frac{x}{\varepsilon}\right) u^{\varepsilon}$ as a function of the micro time step over one macro time step for the convection homogenization problem (4.12). Bottom figure: Time-averaged flux $\bar{j}(x, t)$ as a function of the micro time step.

In summary, we can express the $F$-estimator at time $t$ as

$$
F^{\varepsilon}(U, t)=\tilde{Q}_{\Delta t}\{f(\tilde{u}(\tau)), t \leq \tau \leq t+\Delta t, \tilde{u}(t)=R U\}
$$

where $R$ denotes some reconstruction operator, $\tilde{u}(t)$ is the solution of the compressed microscopic model (possibly over a truncated computational domain) with initial data $\tilde{u}(t)=R U, \tilde{Q}_{\Delta t}$ is the numerical approximation of the compression operator. Typically $\tilde{Q}_{\Delta t}$ has the form

$$
\tilde{Q}_{\Delta t}=Q_{e} Q_{x} Q_{t}
$$


where $Q_{e}, Q_{x}, Q_{t}$ denote the compression operators over the probability, spatial and temporal spaces respectively. Having $F^{\varepsilon}(U, t)$, the macroscopic state variables can be updated using standard ODE solvers. The simplest example of the forward Euler scheme gives

$$
U^{n+1}=U^{n}+\Delta t F^{\varepsilon}\left(U^{n}, t^{n}\right) .
$$

4.2. Simplifications. In many situations, we can simplify the compression techniques further by replacing the compressed microscale solver by explicit macroscale models. The parameters in the macroscale models can be either analytically computed or numerically fitted using the microscale model. This technique is particularly useful for Type A problems which are characterized by the fact that such techniques are applicable over most of the computational domain.

4.2.1. Traditional approach: Explicit macroscopic models. When an explicit macroscopic model is known and known to be valid, the compressed microscopic solver discussed above can be replaced by the traditional approach of using the explicit macroscopic models. This is particularly useful for problems of Type A. In such cases, an adaptive model selection is needed. See, [26].

4.2.2. Semi-empirical methods. This method is a compromise between the explicit macroscopic models and the compressed microscopic models. We assume that the macroscopic model takes a specific form but the coefficients involved might be unknown or change with the environment, and we estimate these coefficients using data obtained from the microscopic models. Such ideas are used extensively as a preprocessing step to calibrate the input data and simulation parameters for macroscopic models. We propose to incorporate such ideas in HMM.

As an example, we discuss the parabolic homogenization problem considered earlier. Even though the macroscale equation for $U=Q u$ might be difficult to obtain explicitly, it must take the form

$$
U_{t}=\nabla \cdot(A(x) \nabla U) .
$$

Our task is to find the tensor $A(x)$. In Figure 4, we plot, for a simple one-dimensional problem with $a(x, y)=2+\sin 2 \pi y$, the computed macroscopic flux (which should be an approximation to $A(x) \nabla U$ ) using the compressed microscopic model as a function of $\nabla U$. We see a clean, straight line whose slope gives an estimate of $A(x)$. Therefore in an actual computation, for each unknown entry of $A$ at a cell boundary, the compressed microscopic solver has to be used only twice at that macroscopic cell boundary to give us an estimate of that entry of $A$.

Other examples of a similar spirit include the dynamic subgrid models of Moin et al. [55] for large eddy simulation of turbulent flows; the work of Vanden-Eijnden on numerical solutions of multiscale stochastic differential equations [78], and model reduction techniques in control theory.

4.2.3. Type A problems. The additional twist for Type A problems is an adaptive procedure that decides on each macroscopic computational element whether the known explicit macroscopic model can be used. We will postpone general discussions on such adaptive procedure to a later publication. 


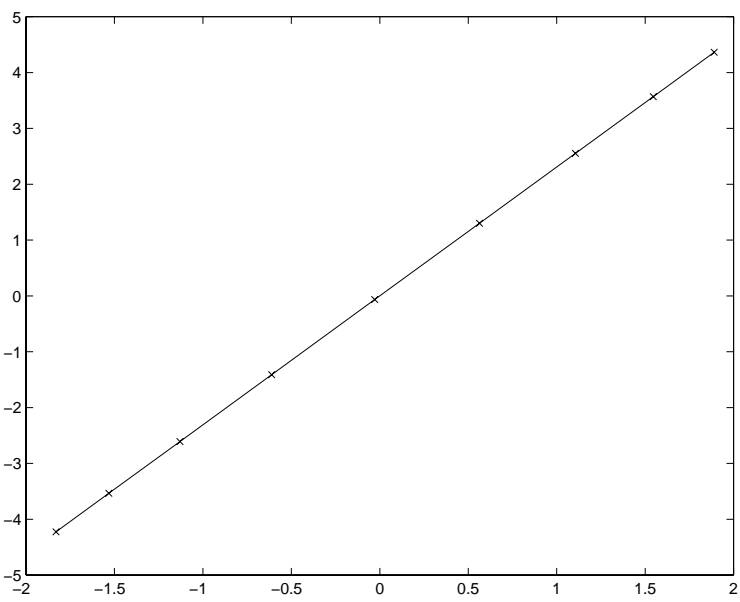

FIG. 4. Empirically computed macroscopic flux as a function of the macroscopic gradient, showing in " $\times$." The line is the exact homogenized flux for the parabolic homogenization problem. This figure illustrates the semi-empirical method. Knowing the form of the macroscopic equation, the unknown coefficients can be empirically estimated with small cost.

It may also happen that near defects or singularities, $t_{R}$ becomes comparable to $t_{M}$. In this case, it may no longer be advantageous to use the compression techniques discussed earlier. Consequently, the compression and reconstruction operators are unnecessary, and the microscopic state should be kept to the next macroscopic time step. This is the case for example in the method proposed in [65] which couples kinetic Monte Carlo and continuum models for epitaxial growth.

4.3. Galerkin formulation. For many problems in applications, it is much more convenient to work with the Galerkin formulation. In this section, we will discuss the extension of HMM to Galerkin formulation. For simplicity we will assume that the fibers satisfy $\mathcal{D}_{x}=\mathcal{D}_{0}$; i.e., they are independent of $x$.

4.3.1. Variational problems. Let $a(u, v)$ be a bilinear form on an appropriate function space $H$ on the microscopic domain $\mathcal{D}=D \times \mathcal{D}_{0}$ satisfying the standard requirements such as continuity and ellipticity. Consider a problem of the form: Find $u \in H$, such that

$$
a(u, v)=(f, v)
$$

for all $v \in H$. Here, $f$ is a given continuous functional on $H$. The version of HMM that we discuss below will be based on a splitting of this problem into two pieces:

1. Let $U$ be a macroscopic state. Denote by $R^{*} U$ the solution of the problem: Find $u$ such that $Q u=U$ and

$$
a(u, v)=0
$$

for all $v$ satisfying the constraint $Q v=0$.

2. Let $\tilde{R}$ be a fixed reconstruction operator, and define a macroscopic bilinear form $A$ by

$$
A(U, V)=a\left(R^{*} U, \tilde{R} V\right)
$$


Then the macroscopic problem is

$$
A(U, V)=(f, \tilde{R} V)
$$

It is easy to see that if $U$ is a solution to (4.20), then $R^{*} U$ is an approximate solution to (4.18) since

$$
a\left(R^{*} U, \tilde{R} V+v\right)=(f, \tilde{R} V) \approx(f, \tilde{R} V+v)
$$

For what follows, it is convenient to take the example of the homogenization problem, for which

$$
a(u, v)=\int_{D} \sum_{i, j} a_{i j}\left(x, \frac{x}{\varepsilon}\right) \frac{\partial u}{\partial x_{i}} \frac{\partial v}{\partial x_{j}} d x
$$

for $u, v \in H=H_{0}^{1}(D)$. In this case $\mathcal{D}_{0}=D$. We emphasize that the methodology applies to general situations. Let $T_{D}$ and $T_{f}$ be finite element triangulations of $D$ and $\mathcal{D}_{0}$ respectively. Standard Galerkin methods based on the microscopic formulation would proceed directly with a finite element space over $T_{D} \times T_{f}$, denoted by $V_{H}^{m}$. In contrast, HMM proceeds in two steps.

Let $V_{H}$ be a standard (e.g. piecewise linear) finite element space over $T_{D}$. We define $Q$ as before: $Q u=U \in V_{H}$, if $\int_{D} \nabla u \nabla V d x=\int_{D} \nabla U \nabla V d x$, for all $V \in V_{H}$. For $U \in V_{H}$, denote by $R^{*} U$ the solution of the problem: Find $u \in V_{H}^{m}, Q u=U$ and

$$
a(u, v)=0
$$

for all $v \in V_{H}^{m}$ satisfying $Q v=0$. The second step is to solve for $U$ from

$$
a\left(R^{*} U, V\right)=(f, V)
$$

for all $V \in V_{H}$.

The key to the efficiency of HMM comes again from the possibility to approximate efficiently $a\left(R^{*} U, V\right)$. The idea is completely analogous to that of the variational problems. For each $H \in T_{D}$ let $x_{K}$ be an interior point of $K$ and let

$$
a_{K}(u, v)=\int_{x_{K}+\varepsilon I} \sum_{i, j} a_{i, j}\left(x, \frac{x}{\varepsilon}\right) \frac{\partial u}{\partial x_{i}} \frac{\partial v}{\partial x_{j}} d x .
$$

Given $U \in V_{H}$, let $u_{K}$ be the solution to the problem

$$
a_{K}(u, v)=0
$$

for all $v \in H^{1}\left(x_{K}+\varepsilon I\right)$, satisfying the constraint that $v$ is periodic with period $\varepsilon I$. $u_{K}$ is also required to satisfy the boundary condition that $u_{K}(x)-U(x)$ is periodic with period $\varepsilon I$. Define

$$
\tilde{A}(U, V)=\sum_{K} \frac{|K|}{\varepsilon^{n}} \int_{x_{K}+\varepsilon I} \sum_{i, j} a_{i, j}\left(x, \frac{x}{\varepsilon}\right) \frac{\partial u_{K}}{\partial x_{i}} \frac{\partial V}{\partial x_{j}} d x
$$

We then approximate $A(U, V)$ by $\tilde{A}(U, V)$. Finally, the macroscale problem is

$$
\tilde{A}(U, V)=(f, V)
$$

for all $V \in V_{H}$.

This method is closely related to the analytic tool of two-scale convergence in homogenization theory $[3,22,56]$. There the idea is to use oscillatory test functions of 
the form $\varphi\left(x, \frac{x}{\varepsilon}\right)$ in order to probe both the macroscopic behavior and the oscillations in the unknowns. Here we split this into two steps: We probe the oscillations in the first step when we compute $u_{K}$, and in the second step we find the approximation to the macroscopic behavior.

4.3.2. Dynamic problems. The set up is similar to that of the variational problem. Consider now a dynamic problem of the form

$$
\frac{d}{d t}(u, v)=a(u, v)
$$

with a corresponding macroscopic model

$$
\frac{d}{d t}(U, V)=A(U, V)
$$

or a time-discretized version

$$
\left(U^{n+1}, V\right)-\left(U^{n}, V\right)=\int_{t^{n}}^{t^{n}+\Delta t} A(U(t), V) d t
$$

for $V \in V_{H}$. To evaluate $A(U, V)$ from microscopic models, we proceed in the following steps:

1. Reconstruction: $\tilde{u}=R U^{n}$.

2. On each element $K$, we solve (4.23) restricted to a small subset of $K \times T_{f}$ with appropriate boundary conditions. For the homogenization problem, we can take this subset to be $x_{K}+\varepsilon I$ and use the periodic boundary condition. From this we can construct an approximation to $A(U(t), V)=a\left(u(t), R^{*} V\right)$ in a similar way as we constructed $\tilde{A}(U, V)$ in the last subsection. We denote this approximation as $\tilde{A}(U(t), V)$. We stop the microscopic solver until $\tilde{A}(U(t), V)$ or its time average reaches a quasistationary value, $A^{*}\left(U^{n}, V\right)$.

3. We let

$$
\left(U^{n+1}, V\right)-\left(U^{n}, V\right)=\Delta t A^{*}\left(U^{n}, V\right)
$$

for $V \in V_{H}$.

In some situations, we can simply use

$$
\left(U^{n+1}, V\right)-\left(U^{n}, V\right)=\Delta t \tilde{A}\left(U^{n}, V\right)
$$

where $\tilde{A}$ was constructed earlier.

4.4. Simple Illustrative examples. Interesting special cases of HMM can already be found in the literature. An immediate first example is the Godunov scheme in gas dynamics [38]. Classical Godunov scheme corresponds to the case when the reconstruction operator is just the identity operator. Nontrivial high order reconstruction operators are used in extensions of the Godunov scheme, such as the MUSCL, PPM and ENO schemes (see for example [51]).

The next interesting example is found in the literature on solving stiff ODEs. In particular, projective methods make use of the time scale separation by combining micro and macro time steps in order to achieve large overall time steps. For details see $[39,49,34]$.

Perhaps the most significant example in the published literature that closely resembles the spirit of the algorithm described above for problems of Type B is the kinetic scheme [79] for gas dynamics. In the form of [79] the kinetic scheme is based 
on the BGK model

$$
f_{t}+(u \cdot \nabla) f=\frac{f^{e q}-f}{\tau}
$$

where $f^{e q}$ is some local equilibrium state, $\tau$ is a small relaxation time. Given the numerical approximation to the local conserved quantities at time $t^{n}$, the kinetic scheme proceeds via the following steps to compute the local conserved quantities at time $t^{n+1}$ :

1. The approximate local equilibrium states $f^{e q}$ and local gas distribution functions are constructed in the neighborhood of the cell boundaries.

2. The BGK equation is solved near the cell boundaries.

3 . The numerical fluxes for the conserved quantities are evaluated from the distribution functions, and the local conserved quantities are updated.

This procedure does not use Euler's equation for gas dynamics. It only makes use of the BGK equation. In connection with HMM, we can define the compression operator $Q$ by

$$
Q f=(\rho, m, E)
$$

where $\rho, m, E$ are respectively the density, momentum, and total energy densities associated with $f$. Designing the reconstruction operator $R$ is the most involved step in [79], which computes corrections to the local Maxwellians in the form of Taylor expansions in order to achieve second order accuracy (see [79] for details).

\section{Stability and Accuracy}

Let $U$ be the numerical solution computed by the heterogeneous multiscale method, $u$ be the exact solution of the microscopic problem, and $Q$ be the compression operator. Our aim was to approximate $Q u$. In this section, we will estimate the error $U-Q u$.

5.1. Variational Problems. Again we will take the classical homogenization problem as an example. But the principle of the analysis applies to more general problems.

Consider the problem

$$
\min _{u \in H_{0}^{1}(D)}\left(\int_{D} \frac{1}{2} \sum_{i, j} a_{i j}\left(x, \frac{x}{\varepsilon}\right) \frac{\partial u}{\partial x_{i}} \frac{\partial u}{\partial x_{j}} d x-\int_{D} f u d x\right)
$$

where $f=f(x)$ is a given smooth function, the $a_{i j}(x, y)$ s are smooth and periodic in $y$ with period $I=[0,1]^{d}$. See section 4.1 .1 for notations. We will assume that the triangulation is quasiregular; i.e., all the triangles are of comparable size, and all angles are bounded uniformly away from 0 . Furthermore, we assume that each triangle contains a cube of size $\varepsilon$.

We will analyze the HMM with $A^{\varepsilon}(U, U)$ approximated by $\tilde{A}(U, U)$, defined in (4.5). Stability comes from the fact that the quadratic form we are dealing with, $\tilde{A}(U, U)$, is still uniformly elliptic if $a^{\varepsilon}(u, u)$ is. Accuracy can be estimated using the first Strang lemma on the approximation of stiffness matrix for finite element methods [17] although for the present problem it is simpler to proceed directly.

Lemma 5.1. Assume that $a^{\varepsilon}(u, u)$ is uniformly $V$-elliptic in the sense that

$$
a^{\varepsilon}(u, u) \geq C_{0} \int_{D}|\nabla u|^{2} d x .
$$


for $u \in H_{0}^{1}(D)$. Then

$$
A^{\varepsilon}(U, U) \geq C_{0} \int_{D}|\nabla U|^{2} d x
$$

for all $U \in V_{H}$.

Proof. From (4.2), we have

$$
\begin{aligned}
A^{\varepsilon}(U, U) & =\min _{u \in H_{0}^{1}(D), Q u=U} a^{\varepsilon}(u, u) \geq \min _{\substack{u \in H_{0}^{1}(D) \\
Q u=U}} C_{0} \int_{D}|\nabla u|^{2} d x \\
& =C_{0} \int_{D}|\nabla U|^{2} d x .
\end{aligned}
$$

LEMMA 5.2. Under the same condition as in Lemma 5.1, we have

$$
\tilde{A}(U, U) \geq C_{0} \int_{D}|\nabla U|^{2} d x
$$

for all $U \in V_{H}$.

Proof. Observe that

$$
\int_{x_{K}+\varepsilon I} \sum_{i, j} a_{i j}\left(x, \frac{x}{\varepsilon}\right) \frac{\partial u_{K}}{\partial x_{i}} \frac{\partial u_{K}}{\partial x_{j}} d x=\min \int_{x_{K}+\varepsilon I} \sum_{i, j} a_{i j}\left(x, \frac{x}{\varepsilon}\right) \frac{\partial u}{\partial x_{i}} \frac{\partial u}{\partial x_{j}} d x
$$

where the minimum is taken over all functions $u$ such that $u-U$ is periodic on $x_{K}+\varepsilon I$. Therefore

$$
\begin{gathered}
\int_{x_{K}+\varepsilon I} \sum_{i j} a_{i, j}\left(x, \frac{x}{\varepsilon}\right) \frac{\partial u_{K}}{\partial x_{i}} \frac{\partial u_{K}}{\partial x_{j}} d x \geq \min C_{0} \int_{x_{K}+\varepsilon I}|\nabla u|^{2} d x \\
=C_{0} \int_{x_{K}+\varepsilon I}|\nabla U|^{2} d x=C_{0} \varepsilon^{d} \cdot|\nabla U|^{2} .
\end{gathered}
$$

This gives the desired result. This completes the proof of Lemma 6.2.

Let $U_{\varepsilon}$ be the solution of

$$
\min _{U \in V_{H}}\left\{A^{\varepsilon}(U, U)-\int_{D} f(x) U(x) d x\right\}
$$

Then it is easy to see that

$$
\left\|U_{\varepsilon}-Q u_{\varepsilon}\right\|_{H^{1}} \leq C H
$$

Let $\tilde{U}_{\varepsilon}$ be the solution of

$$
\min _{U \in V_{H}}\left\{\tilde{A}(U, U)-\int_{D} f(x) U(x) d x\right\}
$$

We will estimate $\left\|U_{\varepsilon}-\tilde{U}_{\varepsilon}\right\|_{H_{0}^{1}(D)}$. The difference is due to the fact that the quadratic form $A^{\varepsilon}$ is approximated by $\tilde{A}$.

We have

$$
C_{0}\left\|\tilde{U}_{\varepsilon}-U_{\varepsilon}\right\|_{H^{1}}^{2} \leq \tilde{A}\left(\tilde{U}_{\varepsilon}-U_{\varepsilon}, \tilde{U}_{\varepsilon}-U_{\varepsilon}\right)=A^{\varepsilon}\left(U_{\varepsilon}, \tilde{U}_{\varepsilon}-U_{\varepsilon}\right)-\tilde{A}\left(U_{\varepsilon}, \tilde{U}_{\varepsilon}-U_{\varepsilon}\right)
$$


Therefore

$$
\left\|\tilde{U}_{\varepsilon}-U_{\varepsilon}\right\|_{H^{1}} \leq \frac{1}{C_{0}} \sup _{V \in V_{H}} \frac{\left|A^{\varepsilon}\left(U_{\varepsilon}, V\right)-\tilde{A}\left(U_{\varepsilon}, V\right)\right|}{\|V\|_{H^{1}}}
$$

The estimate of the right-hand side is carried out in [28], which gives the following theorem.

THEOREM 5.3. Assume that the finite element triangulation is quasi-regular, and that each element of the triangulation contains a cube of size $\varepsilon$, then

$$
\left\|\tilde{U}_{\varepsilon}-Q u_{\varepsilon}\right\|_{H^{1}(D)} \leq C\left(H+\frac{\varepsilon}{H}\right) .
$$

Theorem 6.5 suggests a result of a general nature, namely that the error of HMM for variational problems comes from two sources: the standard local truncation error (here the first term) and the error due to compression (here the second term).

The proof of Theorem 5.3 was complicated by the fact that we were comparing $\tilde{U}_{\varepsilon}$ with $Q u_{\varepsilon}$, therefore we needed to estimate $\tilde{A}-A^{\varepsilon}$. It is simpler to estimate $\tilde{U}_{\varepsilon}-U$, where $U$ is the solution to the homogenized equation. For that purpose, we just have to estimate $\tilde{A}(U, U)-A(U, U)$ where $A(U, U)=\int_{D} \sum_{i, j} A_{i j}(x) \frac{\partial U}{\partial x_{i}} \frac{\partial U}{\partial x_{j}} d x$, and this is a direct consequence of the Lemma 6.5. Therefore we have the following theorem.

THEOREM 5.4. Under the same assumption as in the previous theorem, let $U$ be the solution of

$$
\min _{U \in H_{0}^{1}(D)}\left\{\frac{1}{2} A(U, U)-\int_{D} f U(x) d x\right\}
$$

Then

$$
\left\|\tilde{U}_{\varepsilon}-U\right\|_{H^{1}(D)} \leq C H
$$

Having $\tilde{U}$, we can construct approximations to $u^{\varepsilon}$. This is done in [24, 28].

5.2. Dynamic Problems of Type B. For concreteness we will discuss the case when the macroscopic model is a differential equation of the form $U_{t}=F(U)$, and HMM takes the form

$$
U_{j}^{n+1}=U_{j}^{n}+\Delta t F_{j}^{\varepsilon}\left(U^{n}\right) .
$$

We will estimate the error between $\left\{U_{j}^{n}\right\}$ and some suitably chosen numerical scheme for the macroscopic model. Let us write this numerical scheme in the form

$$
\bar{U}_{j}^{n+1}=\bar{U}_{j}^{n}+\Delta t \bar{F}_{j}\left(\bar{U}^{n}\right) .
$$

Let $E_{j}^{n}=U_{j}^{n}-\bar{U}_{j}^{n}$. Then

$$
E_{j}^{n+1}=E_{j}^{n}+\Delta t\left(\bar{F}_{j}\left(U^{n}\right)-\bar{F}_{j}\left(\bar{U}^{n}\right)\right)+\Delta t\left(F_{j}^{\varepsilon}\left(U^{n}\right)-\bar{F}_{j}\left(U^{n}\right)\right) .
$$

$\bar{F}$ should be chosen so that it is stable and minimizes $F_{j}^{\varepsilon}-\bar{F}_{j}$. For this purpose, we define the Generalized Godunov Scheme (GGS), which is obtained by replacing the microscopic solver in HMM by the exact macroscopic solver. The precise definition is not important. We just have to identify one particular macroscopic scheme that is both stable and close to HMM. Examples of GGS will be given below. 
For nonlinear problems, we have to be careful about the notion of stability. For problems with smooth numerical solutions for which divided differences of suitable orders are uniformly bounded, Strang showed that $L^{2}$ stability of the linearized equation is sufficient for getting the full accuracy of the nonlinear problem [72]. For multiscale problems, even though we are interested in macroscopic variables which are typically averaged quantities, small scale fluctuations still exist at small amplitudes, and we have to specify more carefully the class of numerical solutions that are allowed when we define stability.

Fix a macroscopic time $T$. Let $K$ be a class of discrete functions defined on the numerical grid. For example in Strang's theorem, $K=\left\{\left\{U_{j}^{n}\right\}\right.$, $\left\|D_{H}^{\alpha} U_{j}^{n}\right\| \leq C_{\alpha}$ for $\alpha \leq N$, and $n \Delta t \leq T\}$, where $N$ is an integer depending on $d$, the spatial dimension; $C_{\alpha}$ is independent of $\Delta t$; and $H, D_{H}^{\alpha}$ is the divided difference operator of order $\alpha$. For our problem, $K$ will usually be chosen to consist of discrete functions which have bounded divided differences up to some order, and which are close to the exact solution of the macroscopic model.

Definition. The GGS is said to be $(K, \delta)$ stable if

$$
\left\|\Pi_{n=k}^{\ell}\left(I+\Delta t \int_{0}^{1} \nabla \bar{F}\left(U^{n}+\theta\left(V^{n}-U^{n}\right)\right) d \theta\right)\right\| \leq \text { Constant }
$$

for all $k, \ell$ such that $0 \leq k \leq \ell \leq\left[\frac{T}{\Delta t}\right]$, and all $\left\{U^{n}\right\},\left\{V^{n}\right\}$ such that $\left\{U^{n}\right\},\left\{V^{n}\right\} \in K$, and

$$
\left\|U^{n}-V^{n}\right\| \leq \delta
$$

if $n \Delta t \leq T$. The constant depends only on $T$.

TheOrem 5.5. Assume that

1. $G G S$ is $(K, \delta)$-stable

2. $\left\{\bar{U}^{n}\right\},\left\{U^{n}\right\} \in K$

3. $\max _{n \leq \frac{T}{\Delta t}}\left\|\bar{F}\left(U^{n}\right)-F^{\varepsilon}\left(U^{n}\right)\right\| \rightarrow 0$ and $\left\|\bar{U}^{0}-U^{0}\right\| \rightarrow 0$ as $\varepsilon \rightarrow 0, \Delta t, H \rightarrow 0$.

Then there exist $\varepsilon_{0}, \Delta t_{0}, H_{0}$, such that if $\varepsilon<\varepsilon_{0}, \Delta t<\Delta t_{0}, H<H_{0}, n \Delta t \leq T$

$$
\left\|\bar{U}^{n}-U^{n}\right\| \leq C\left(\left\|\bar{U}^{0}-U^{0}\right\|+\max _{0 \leq k \leq \frac{T}{\Delta t}}\left\|\bar{F}\left(U^{k}\right)-F^{\varepsilon}\left(U^{k}\right)\right\|\right) .
$$

Proof. First let us assume that

$$
\left\|\bar{U}^{n}-U^{n}\right\| \leq \delta .
$$

Let $\bar{S}^{n}=I+\Delta t \int_{0}^{1} \nabla \bar{F}\left(\bar{U}^{n}+\theta\left(U^{n}-\bar{U}^{n}\right)\right) d \theta$. Then from (5.3) we have

$$
E^{n}=\left(\Pi_{\ell=0}^{n-1} \bar{S}^{\ell}\right) E^{0}+\Delta t \sum_{k=0}^{n-1}\left(\Pi_{\ell=k}^{n-1} \bar{S}^{\ell}\right)\left(F^{\varepsilon}\left(U^{k}\right)-\bar{F}\left(U^{k}\right)\right) .
$$

Hence, using $(K, \delta)$ stability,

$$
\begin{aligned}
\left\|E^{n}\right\| & \leq C_{0}\left\|E^{0}\right\|+\Delta t \sum_{k=0}^{n-1} C_{0}\left\|F^{\varepsilon}\left(U^{k}\right)-\bar{F}\left(U^{k}\right)\right\| \\
& \leq C_{0}\left\|E^{0}\right\|+T C_{0} \max _{k \leq \frac{T}{\Delta t}}\left\|F^{\varepsilon}\left(U^{k}\right)-\bar{F}\left(U^{k}\right)\right\| .
\end{aligned}
$$


Now we will show that the assumption that $\left\|\bar{U}^{n}-U^{n}\right\| \leq \delta$ will not be violated for $n \Delta t \leq T$. Since $\left\|\bar{U}^{0}-U^{0}\right\| \rightarrow 0$, as $\varepsilon \rightarrow 0, \Delta t, H \rightarrow 0$, we have $\left\|\bar{U}^{0}-U^{0}\right\| \leq \delta$. Assume that $\left\|\bar{U}^{k}-U^{k}\right\| \leq \delta$ for all $k \leq n \leq \frac{T}{\Delta t}$. If $n+1 \leq \frac{T}{\Delta t}$, then we can use the $(K, \delta)$ stability to get

$$
\left\|E^{n+1}\right\| \leq C_{0}\left\|E^{0}\right\|+T C_{0} \max _{k \leq \frac{t}{\Delta t}}\left\|F^{\varepsilon}\left(U^{k}\right)-\bar{F}\left(U^{k}\right)\right\| .
$$

From the third assumption in the statement of the theorem, there exist $\varepsilon_{0}, \Delta t_{0}, H_{0}$ such that if $\varepsilon<\varepsilon_{0}, \Delta t<\Delta t_{0}, H<H_{0}$, then the right-hand side of the above inequality is less that $\delta$. Hence $\left\|E^{n+1}\right\| \leq \delta$ and by induction this proves that $\left\|E^{n}\right\| \leq \delta$ if $n \leq \frac{T}{\Delta t}$ and $\varepsilon<\varepsilon_{0}, \Delta t<\Delta t_{0}, H<H_{0}$. This completes the proof of the theorem.

Theorem 5.8 does not guarantee that $\left\{U^{n}\right\}$ is a good approximation to the true macroscopic behavior of the system. For this to happen it is important to assume that the GGS is consistent. Consider the following illustrative example for solving the parabolic homogenization problem (4.9). The proposed method consists of the following steps.

1. Given $\left\{U_{j}^{n}\right\}$, let $R U^{n}(x)$ be the piecewise linear reconstruction $R U^{n}(x)=$ $U_{j}^{n}+\frac{U_{j+1}^{n}-U_{j-1}^{n}}{2 H}\left(x-x_{j}\right), x_{j-\frac{1}{2}} \leq x<x_{j+\frac{1}{2}}$.

2. On $\left[x_{j}, x_{j}+\varepsilon\right]$, solve (4.9) with initial data $u\left(x, t^{n}\right)=R U^{n}(x)$ and the boundary condition that $u(x, t)-R U^{n}(x)$ is periodic.

3. Let $F_{j}^{n}$ be the average of $\nabla \cdot\left(a\left(x, \frac{x}{\varepsilon}\right) \nabla u^{\varepsilon}\left(x, t^{n+1}\right)\right)$ over $\left[x_{j}, x_{j}+\varepsilon\right]$.

4. $U_{j}^{n+1}=U_{j}^{n}+\Delta t F_{j}^{n}$.

The corresponding GGS is the same except we replace the microscopic model in Steps 2 and 3 by the homogenized equation.

This algorithm is unlikely to converge to the true macroscopic behavior described by the homogenized equation since the GGS itself is not consistent with the homogenized equation. In fact it is easy to check that the GGS produces $U_{t}=0$. For consistency, the reconstruction has to be at least piecewise quadratic.

Corollary 5.6. Assume that the conditions in Theorem 5.8 hold, and let $\bar{U}^{0}=U^{0}$. Then

$$
\left\|U^{n}-Q u\left(\cdot, t^{n}\right)\right\| \leq C\left(\left\|\bar{U}^{n}-Q u\left(\cdot, t^{n}\right)\right\|+\max _{0 \leq k \leq \frac{T}{\Delta t}}\left\|\bar{F}\left(U^{k}\right)-F^{\varepsilon}\left(U^{k}\right)\right\|\right) .
$$

The error has two contributions: The first term is the standard local truncation error of the macroscopic scheme and the second term is the error due to compression.

The compression error also has two contributions: error due to compression in time and error due to compression in space. Here we will study the error due to compression in time.

In many situations, the macroscopic model takes the form

$$
F(U)=Q f\left(R^{*} U\right)
$$

where $R^{*} U$ is the microscopic "local equilibrium" state associated with $U$. Recall that $F^{\varepsilon}(U, t)$ has the form

$$
\begin{aligned}
F^{\varepsilon}(U, t) & =\tilde{Q}_{\Delta t}\{f(\tilde{u}(\tau)), t \leq \tau \leq t+\Delta t, \tilde{u}(t)=R U\} \\
\tilde{Q}_{\Delta t} & =Q_{e} Q_{x} Q_{t} .
\end{aligned}
$$

For simplicity, let us assume that $\tilde{u}(\tau)=u(\tau)$; i.e., the microscopic solver is exact. 
Observe that if we replace $\tilde{u}(\tau)$ by $R^{*} U$, then

$$
Q_{t}\left\{f\left(R^{*} U\right)\right\}=f\left(R^{*} U\right) .
$$

Let

$$
\tilde{Q} f\left(R^{*} U\right)=Q_{e} Q_{x} f\left(R^{*} U\right)
$$

then

$$
\begin{gathered}
F(U)-F^{\varepsilon}(U, t)=Q f\left(R^{*} U\right)-\tilde{Q} f\left(R^{*} U\right) \\
+\tilde{Q} f\left(R^{*} U\right)-\tilde{Q}_{\Delta t}\{f(u(\tau)), t \leq \tau \leq t+\Delta t, u(t)=R U\} .
\end{gathered}
$$

In the following, we will consider special cases for which $\tilde{Q}=Q$. Then estimating $F(U)-F^{\varepsilon}(U, t)$ reduces to estimating

$$
Q\left(f\left(R^{*} U\right)-Q_{t} f\{u(\tau), t \leq \tau \leq t+\Delta t, u(t)=R U\}\right) .
$$

This clearly depends on the rate of relaxation to local equilibrium. It is relatively easy to calibrate this term when strong relaxation takes place.

LеммA 5.7. Let $u(\tau)$ be the solution of the microscale problem with initial data $u(0)=R U$. Assume that strong relaxation takes place in the sense that

$$
\begin{gathered}
\left|u(\tau)-R^{*} U\right| \leq C e^{-\frac{\tau}{\delta(\varepsilon)}} \\
\left|Q f(u(\tau))-Q f\left(R^{*} U\right)\right| \leq C e^{-\frac{\tau}{\delta(\varepsilon)}}
\end{gathered}
$$

and let

$$
\left.Q_{t}\{f(u(\tau)), t \leq \tau \leq t+\Delta t, u(t)=R U)\right\}=f(u(t+\alpha \Delta t))
$$

then

$$
\left|F(U)-F^{\varepsilon}(U, t)\right| \leq C e^{-\frac{\alpha \Delta t}{\delta(\varepsilon)}}
$$

Typically (5.6) and (5.7) holds if there is a spectral gap. $\delta(\varepsilon)$ is the relaxation time which goes to zero as $\varepsilon$ goes to zero.

The case of weak relaxation is more subtle since non trivial time averaging is necessary in order to guarantee convergence. We will postpone a discussion of the general theory to [23].

For what follows, we will concentrate on linear problems of the type

$$
U_{t}+\nabla \cdot J=0
$$

for which the notion of standard linear stability suffices. In this case, it is natural to use the finite volume method as the underlying macroscopic scheme. Instead of estimating forces, we estimate macroscopic fluxes at the cell boundaries.

We will compare the solutions of the HMM, expressed in the form

$$
\frac{U_{j}^{n+1}-U_{j}^{n}}{\Delta t}+\frac{J_{j+\frac{1}{2}}^{\varepsilon}\left(U^{n}\right)-J_{j-\frac{1}{2}}^{\varepsilon}\left(U^{n}\right)}{H}=0
$$

where $J_{j+\frac{1}{2}}^{\varepsilon}$ is the numerical flux computed via HMM at the cell boundary at $x_{j+\frac{1}{2}}$, 
with that of an associated macroscopic scheme

$$
\frac{\bar{U}_{j}^{n+1}-\bar{U}_{j}^{n}}{\Delta t}+\frac{\bar{J}_{j+\frac{1}{2}}\left(\bar{U}^{n}\right)-\bar{J}_{j-\frac{1}{2}}\left(\bar{U}^{n}\right)}{\Delta x}=0 .
$$

Let $E_{j}^{n}=U_{j}^{n}-\bar{U}_{j}^{n}$. Then we have

$$
\begin{aligned}
\frac{E_{j}^{n+1}-E_{j}^{n}}{\Delta t} & +\frac{\bar{J}_{j+\frac{1}{2}}\left(E^{n}\right)-\bar{J}_{j-\frac{1}{2}}\left(E^{n}\right)}{\Delta x} \\
& +\frac{1}{\Delta x}\left\{\left(J_{j+\frac{1}{2}}^{\varepsilon}\left(U^{n}\right)-\bar{J}_{j+\frac{1}{2}}\left(U^{n}\right)\right)-\left(J_{j-\frac{1}{2}}^{\varepsilon}\left(U^{n}\right)-\bar{J}_{j-\frac{1}{2}}\left(U^{n}\right)\right)\right\}=0 .
\end{aligned}
$$

Lemma 5.8. Assume that the GGS is stable. Then we have

$$
\left|E_{j}^{n}\right| \leq C_{0} \max _{U}\left|\frac{1}{\Delta x}\left\{\left(J_{j+\frac{1}{2}}^{\varepsilon}(U)-\bar{J}_{j+\frac{1}{2}}(U)\right)-\left(J_{j-\frac{1}{2}}^{\varepsilon}(U)-\bar{J}_{j-\frac{1}{2}}(U)\right)\right\}\right|
$$

In some situations, the quantity $H_{j+\frac{1}{2}}^{\varepsilon}(U)=J_{j+\frac{1}{2}}^{\varepsilon}(U)-\bar{J}_{j+\frac{1}{2}}(U)$ is smoothly varying in $j$, therefore the divided difference of $H^{\varepsilon}(U)$ is comparable in magnitude with $H^{\varepsilon}(U)$ itself.

Our task is now reduced to estimating

$$
\max _{U} \frac{1}{\Delta x}\left|H_{j+\frac{1}{2}}^{\varepsilon}(U)-H_{j-\frac{1}{2}}^{\varepsilon}(U)\right| .
$$

We will discuss this using several concrete examples.

Our first example is the advection equation

$$
u_{t}^{\varepsilon}+\nabla \cdot\left(a\left(\frac{x}{\varepsilon}\right) u^{\varepsilon}\right)=0
$$

in one dimension, where $a(y)$ is assumed to be smooth and strictly positive. We will consider a simplified situation in which the microscopic solver is exact, and $J^{\varepsilon}$ is computed as the averages over a small interval of size $\alpha \Delta t$

$$
J_{j+\frac{1}{2}}^{\varepsilon}=\frac{1}{\alpha \Delta t} \int_{0}^{\alpha \Delta t} a\left(\frac{x_{j+\frac{1}{2}}}{\varepsilon}\right) u^{\varepsilon}\left(x_{j+\frac{1}{2}}, t\right) d t
$$

where $u^{\varepsilon}(x, t)$ is the exact solution of (5.14) with the reconstruction $u(x, 0)=U_{j}$ for $x<x_{j+\frac{1}{2}} \cdot u(x, 0)=U_{j+1}$ for $x>x_{j+\frac{1}{2}}$.

For this particular problem, the macroscopic equation is simply

$$
U_{t}+\nabla \cdot(A U)=0
$$

where $A=\left(\int_{0}^{1} \frac{1}{a(y)} d y\right)^{-1}$. The GGS is the upwind scheme: $\bar{J}_{j+\frac{1}{2}}(U)=A U_{j-1}$. The stability condition is $\frac{\Delta t}{\Delta x} A<1$.

To compute $J_{j+\frac{1}{2}}^{\varepsilon}$, let $v^{\varepsilon}$ be defined as $v_{x}^{\varepsilon}=u^{\varepsilon}$, and $v^{\varepsilon}$ solves the equation

$$
v_{t}^{\varepsilon}+a^{\varepsilon}(x) v_{x}^{\varepsilon}=0
$$

with initial data $v^{\varepsilon}(x, 0)=U_{j}\left(x-x_{j+\frac{1}{2}}\right)$ for $x<x_{j+\frac{1}{2}}$ and $v^{\varepsilon}(x, 0)=U_{j+1}\left(x-x_{j+\frac{1}{2}}\right)$ for $x>x_{j+\frac{1}{2}}$. Here $a^{\varepsilon}(x)=a\left(\frac{x}{\varepsilon}\right)$. This equation is easily solved by the method of 
characteristics. Let $X(y, t)$ be the solution of

$$
\frac{d X(y, t)}{d t}=a^{\varepsilon}(X(y, t)), X(y, 0)=y
$$

and $y(x, t)$ be the inverse function of $X(y, t)$ (with $t$ fixed), $X(y(x, t), t)=x$. Then $v^{\varepsilon}(x, t)=U_{j} y(x, t)$. Moreover

$$
\begin{aligned}
a^{\varepsilon}(x) u^{\varepsilon} & =a^{\varepsilon}(x) v_{x}^{\varepsilon}=a^{\varepsilon}(x) U_{j} \frac{\partial y}{\partial x} \\
& =a^{\varepsilon}(x) U_{j} \frac{a^{\varepsilon}(y)}{a^{\varepsilon}(x)}=a^{\varepsilon}(y) U_{j} .
\end{aligned}
$$

Therefore we have

$$
\begin{aligned}
J_{j+\frac{1}{2}}^{\varepsilon} & =\frac{1}{\alpha \Delta t} \int_{0}^{\alpha \Delta t} a^{\varepsilon}\left(x_{j+\frac{1}{2}}\right) u^{\varepsilon}\left(x_{j+\frac{1}{2}}, t\right) d t \\
& =\frac{1}{\alpha \Delta t} U_{j} \int_{0}^{\alpha \Delta t} a^{\varepsilon}\left(y\left(x_{j+\frac{1}{2}}, t\right)\right) d t \\
& =\frac{1}{\alpha \Delta t} U_{j} \int_{0}^{\alpha \Delta t} a^{\varepsilon}(y) d y \cdot\left(\frac{d t}{d y}\right) \\
& =-\frac{1}{\alpha \Delta t} U_{j} \int_{0}^{\alpha \Delta t} d y
\end{aligned}
$$

since $\frac{d t}{d y}=-\frac{1}{a^{\varepsilon}(y)}$. Hence,

$$
J_{j+\frac{1}{2}}^{\varepsilon}=U_{j} \frac{y\left(x_{j+\frac{1}{2}}, 0\right)-y\left(x_{j+\frac{1}{2}}, \alpha \Delta t\right)}{\alpha \Delta t}=U_{j} \frac{x_{j+\frac{1}{2}}-y\left(x_{j+\frac{1}{2}}, \alpha \Delta t\right)}{\alpha \Delta t} .
$$

From (5.18), it is easy to see that

$$
\left(x_{j+\frac{1}{2}}-y\left(x_{j+\frac{1}{2}}, \alpha \Delta t\right)\right) \int_{0}^{1} \frac{1}{a\left(x_{j+\frac{1}{2}}, y\right)} d y+O(\varepsilon)=\alpha \Delta t .
$$

Therefore,

$$
J_{j+\frac{1}{2}}^{\varepsilon}=U_{j}\left(\int_{0}^{1} \frac{1}{a\left(x_{j+\frac{1}{2}}, y\right)} d y\right)^{-1}+O\left(\frac{\varepsilon}{\alpha \Delta t}\right)=U_{j} A\left(x_{j+\frac{1}{2}}\right)+O\left(\frac{\varepsilon}{\alpha \Delta t}\right) .
$$

Hence,

$$
\frac{1}{\Delta x}\left|J_{j+\frac{1}{2}}^{\varepsilon}-J_{j-\frac{1}{2}}^{\varepsilon}\right| \leq C \frac{\varepsilon}{\alpha \Delta t \Delta x}
$$

From Lemma 5.9, we have

$$
\left|U_{j}^{n}-\bar{U}_{j}^{n}\right| \leq C \frac{\varepsilon}{\alpha \Delta t \Delta x}
$$

which in turn gives

$$
\left|U_{j}^{n}-(Q u)\left(x_{j}, t^{n}\right)\right| \leq C\left(\frac{\varepsilon}{\alpha \Delta t \Delta x}+\Delta x\right) .
$$

This estimate is not optimal due to (5.24). 
For our second example, we take

$$
u_{t}^{\varepsilon}=\nabla \cdot\left(a\left(x, \frac{x}{\varepsilon}\right) \nabla u^{\varepsilon}\right)
$$

in one dimension with boundary condition $u^{\varepsilon}(0, t)=u^{\varepsilon}(1, t)=0$.

For simplicity we will neglect possible technical difficulty at the boundary. This can be assured by choosing $\varepsilon$ in the form $\varepsilon=1 / N$ where $N$ is an integer. Our argument also applies to the general case, with slight modification.

Let

$$
\hat{u}^{\varepsilon}(x, t)=u_{0}(x, t)+\varepsilon u_{1}\left(x, \frac{x}{\varepsilon}, t, \frac{t}{\varepsilon^{2}}\right)+\varepsilon^{2} u_{2}\left(x, \frac{x}{\varepsilon}, t, \frac{t}{\varepsilon^{2}}\right)
$$

where $u_{0}$ satisfies the homogenized equation

$$
\partial_{t} u_{0}=\nabla \cdot\left(A(x) \nabla u_{0}\right)
$$

with $A(x)=\left(\int_{0}^{1} \frac{1}{a(x, y)} d y\right)^{-1}, u_{1}, u_{2}, \ldots$ satisfies, for fixed $(x, t)$

$$
\begin{aligned}
& \partial_{\tau} u_{1}-\nabla_{y}\left(a(x, y)\left(\nabla_{y} u_{1}+\nabla_{x} u_{0}\right)\right)=0 \\
& \partial_{\tau} u_{2}-\nabla_{y}\left(a(x, y)\left(\nabla_{y} u_{2}+\nabla_{x} u_{1}\right)\right)=\nabla_{x}\left(a(x, y)\left(\nabla_{y} u_{1}+\nabla_{x} u_{0}\right)\right)-\nabla_{x}\left(A(x) \nabla_{x} u_{0}\right)
\end{aligned}
$$

etc. Consider the family of operators defined by

$$
L^{x} u(y)=-\nabla_{y}\left(a(x, y) \nabla_{y} u(y)\right) .
$$

with periodic boundary condition. For every fixed $x$ it has a zero eigenvalue and other eigenvalues are positive. Let $\lambda(x)$ be its smallest positive eigenvalue, and let $\tilde{u}_{1}(x, y, t), \tilde{u}_{2}(x, y, t) \cdots$ be the solutions of

$$
\begin{aligned}
& \nabla_{y}\left(a(x, y)\left(\nabla_{y} \tilde{u}_{1}+\nabla_{x} u_{0}\right)\right)=0 \\
& \nabla_{y}\left(a(x, y)\left(\nabla_{y} \tilde{u}_{2}+\nabla_{x} u_{1}\right)\right)=\nabla_{x}\left(A(x) \nabla_{x} u_{0}\right)-\nabla_{x}\left(a(x, y)\left(\nabla_{y} \tilde{u}_{1}+\nabla_{x} u_{0}\right)\right) .
\end{aligned}
$$

In general, their averages with respect to $y$ are determined by the slow equations of $\tilde{u}_{1}, \tilde{u}_{2}$ obtained from higher order terms in the hierarchy, see [8]. Here we can take their averages to be 0 . Then we have

$$
\begin{aligned}
& \left|u_{1}(x, y, t, \tau)-\tilde{u}_{1}(x, y, t)\right| \leq C e^{-\lambda(x) \tau} \\
& \left|u_{2}(x, y, t, \tau)-\tilde{u}_{2}(x, y, t)\right| \leq C e^{-\lambda(x) \tau} .
\end{aligned}
$$

Now let

$$
\begin{aligned}
& \tilde{J}^{\varepsilon}(x, t)=a\left(x, \frac{x}{\varepsilon}\right) \partial_{x}\left(u_{0}(x)+\varepsilon \tilde{u}_{1}\left(x, \frac{x}{\varepsilon}, t\right)+\varepsilon^{2} \tilde{u}_{2}\left(x, \frac{x}{\varepsilon}, t\right)\right) \\
& \hat{J}^{\varepsilon}(x, t)=a\left(x, \frac{x}{\varepsilon}\right) \partial_{x} \hat{u}^{\varepsilon}, \quad J^{\varepsilon}(x, t)=a\left(x, \frac{x}{\varepsilon}\right) \partial_{x} u^{\varepsilon}
\end{aligned}
$$

we then have

$$
\begin{gathered}
\left|\hat{J}^{\varepsilon}(x, t)-\tilde{J}^{\varepsilon}(x, t)\right| \leq C e^{-\frac{\lambda t}{\varepsilon^{2}}} \\
\left|\hat{J}^{\varepsilon}(x, t)-J^{\varepsilon}(x, t)\right| \leq C \varepsilon
\end{gathered}
$$


with $\lambda=\min _{x} \lambda(x)$ and the constant $C$ only depends on $u_{0}$ and $\Delta x . C$ takes the form $C_{0} /(\Delta x)^{k}$ where $k$ depends on the smoothness of the reconstruction. In one dimension, we have

$$
a(x, y)\left(\partial_{x} u_{0}+\partial_{y} u_{1}\right)=A(x) \partial_{x} u_{0}=J(x, t) .
$$

Hence, $\tilde{J}^{\varepsilon}(x, t)-J(x, t)$ can be expressed as

$$
\tilde{J}^{\varepsilon}(x, t)-J(x, t)=\varepsilon f_{1}\left(x, \frac{x}{\varepsilon}, t\right)+\varepsilon^{2} f_{2}\left(x, \frac{x}{\varepsilon}, t\right) .
$$

Therefore we have

$$
\left|\frac{1}{\Delta x}\left(J^{\varepsilon}\left(x_{j+\frac{1}{2}}, t\right)-J^{\varepsilon}\left(x_{j-\frac{1}{2}}, t\right)\right)-\frac{1}{\Delta x}\left(J\left(x_{j+\frac{1}{2}}, t\right)-J\left(x_{j-\frac{1}{2}}, t\right)\right)\right| \leq \frac{C_{0}}{(\Delta x)^{k}}\left(\varepsilon+e^{-\frac{\lambda t}{\varepsilon^{2}}}\right) .
$$

Going back to the HMM, if we assume that the microscopic solver is exact, and the flux-estimator is given by $J^{\varepsilon}(x, \alpha \Delta t)$ where $\alpha \in(0,1)$, then the GGS is given by

$$
\frac{\bar{U}_{j}^{n+1}-\bar{U}_{j}^{n}}{\Delta t}=-\frac{1}{\Delta x}\left(J\left(x_{j+\frac{1}{2}}, \alpha \Delta t\right)-J\left(x_{j-\frac{1}{2}}, \alpha \Delta t\right)\right)
$$

where $J(x, t)$ is obtained from exact solution of the homogenized equation with the same reconstruction as in the HMM, and we finally obtain an estimate of the type

$$
\left|\bar{U}_{j}^{n}-U_{j}^{n}\right| \leq \frac{C_{0}}{(\Delta x)^{k+1}}\left(\varepsilon+e^{-\frac{\lambda \alpha \Delta t}{\varepsilon^{2}}}\right)
$$

where $k$ depends on the regularity of the reconstruction.

This analysis cannot be extended to high dimension since (5.27) ceases to be valid in high dimension. In fact, HMM in the flux form may not converge in high dimension. This is discussed further in Section 5.4.

For our third example, we consider the case of an ensemble of independent random walkers on the line. This example serves the purpose of illustrating the effect of different reconstructions on the rate of relaxation. The macroscopic equation for the average density of the walkers is simply the heat equation. On a macroscopic grid, the HMM can be formulated as

$$
\frac{U_{j}^{n+1}-U_{j}^{n}}{\Delta t}+\frac{J_{j+\frac{1}{2}}^{n}-J_{j-\frac{1}{2}}^{n}}{\Delta x}=0
$$

where $J_{j+\frac{1}{2}}^{n}$ is computed by ensemble averaging (over $N$ independent copies) of the empirical flux across the boundary at $x_{j+\frac{1}{2}}$ obtained by placing particles according to some reconstructed distributions. The empirical flux is computed as $N_{+}(\alpha \Delta t)-$ $N_{-}(\alpha \Delta t)$, where $N_{ \pm}(\alpha \Delta t)$ denotes the number of particles crossing the cell boundary at $x_{j+\frac{1}{2}}$ from the right/left respectively, up to time $\alpha \Delta t$.

For simplicity, we will let $x_{j+\frac{1}{2}}=0$, and replace the random walks by their continuous counterpart, the Brownian paths. Denote by $y_{k}, k=-K,-K+1, \ldots, K$ the initial position of the Brownian particles. At later time, the positions of the Brownian particles are at $x_{k}^{\ell}(t)=y_{k}+w_{k}^{\ell}(t), \ell=1, \ldots N$. We will consider two different cases.

CASE 1. $w_{k}^{\ell}(t)=w^{\ell}(t)$ where $\left\{w^{\ell}(\cdot)\right\}$ are independent Brownian paths.

CAsE 2. $\left\{w_{k}^{\ell}(\cdot)\right\}$ are independent Brownian paths for $\ell=1, \cdots N, k=-K,-K+$ $1, \ldots, K$. 
Let $N_{k}(t)$ be the number of particles starting at $y_{k}$ that cross 0 before time $t$. For $k>0$, let

$$
\chi_{k, \ell}(t, w)=1_{\left\{\min _{0 \leq s \leq t} w_{k}^{\ell}(s)<-y_{k}\right\}}
$$

where $1_{B}$ denotes the indicator function of the set $B, w$ denotes the random path. Then

$$
N_{k}(t)=\sum_{\ell} \chi_{k, \ell}(t, w)
$$

Using the reflection principle, we have

$$
\begin{aligned}
\mathrm{E} \chi_{k, \ell}(t, w) & =\operatorname{Prob}\left\{\min _{0 \leq s \leq t} w_{k}^{\ell}(s)<-y_{k}\right\} \\
& =2 \operatorname{Prob}\left\{w(t)<-y_{k}\right\} \\
& =2 \int_{-\infty}^{-y_{k}} H_{t}(\lambda) d \lambda=f_{t}\left(y_{k}\right)
\end{aligned}
$$

where $H_{t}(\lambda)=\frac{1}{\sqrt{4 \pi t}} e^{-\frac{\lambda^{2}}{4 t}}$. The last equality defines $f_{t}(y)$. Hence,

$$
\mathrm{E} N_{k}(t)=\sum_{\ell} \mathrm{E} \chi_{k, \ell}(t, w)=N f_{t}\left(y_{k}\right)
$$

If we denote by $N_{+}(t)$ the total number of particles that cross $x=0$ from the right before time $t$, we have

$$
\mathrm{E} \frac{N_{+}(t)}{N}=\sum_{k>0} f_{t}\left(y_{k}\right) .
$$

Consider first the case when $\left\{y_{k}\right\}$ is distributed as $y_{k}=\frac{k \cdot \delta x}{U_{+}}$for $k>0$, and for $k<0$, $y_{k}=\frac{k \delta x}{U_{-}}$. Here $U_{+}=U_{j+1}, U_{-}=U_{j}$. This corresponds to a piecewise constant reconstruction. Let $\Delta x=K \delta x$.

$$
\begin{aligned}
\mathrm{E} \frac{N_{+}(t)}{N} & =\sum_{k \geq 0} f_{t}\left(\frac{k \delta x}{U_{+}}\right)=\frac{U_{+}}{\delta x}\left(\int_{0}^{\Delta x} f_{t}(y) d y+O(1)\right) \\
& =\frac{U_{+}}{\delta x}\left\{\sqrt{\frac{t}{\pi}}\left(1-e^{-\frac{(\Delta x)^{2}}{4 t}}\right)+\Delta x \operatorname{erf}\left(\frac{\Delta x}{\sqrt{2 t}}\right)\right\}
\end{aligned}
$$

where $\operatorname{erf}(y)=\frac{1}{\sqrt{2 \pi}} \int_{y}^{\infty} e^{-\frac{x^{2}}{2}} d x$. As $K \rightarrow \infty$, we have

$$
\mathrm{E} \frac{N_{+}(t)}{N} \rightarrow \sqrt{\frac{t}{\pi}} \frac{U_{+}}{\delta x} .
$$

Similarly for $k<0$

$$
\mathrm{E} \frac{N_{-}(t)}{N} \rightarrow \sqrt{\frac{t}{\pi}} \frac{U_{-}}{\delta x}
$$


where $N_{-}(t)$ is the total number of particles that cross $x=0$ from the left. The ensemble averaged flux is

$$
J(t)=\mathrm{E} \frac{N_{+}(t)-N_{-}(t)}{N}=\sqrt{\frac{t}{\pi}} \frac{1}{\delta x}\left(U_{+}-U_{-}\right) .
$$

This has a square root behavior in $t$, instead of linear. As expected, the rate of crossing 0 slows down as time increases. To compute the variance, let us first consider Case 2. Notice that

$$
\begin{aligned}
\mathrm{E} N_{k}^{2}(t)-\left(\mathrm{E} N_{k}(t)\right)^{2} & =\sum_{\ell, \ell^{\prime}} \mathrm{E} \chi_{k, \ell} \chi_{k, \ell^{\prime}}-\left(\sum_{\ell} \mathrm{E} \chi_{k, \ell}\right)^{2} \\
& =\sum_{\ell} \mathrm{E} \chi_{k, \ell}+\sum_{\ell \neq \ell^{\prime}} \mathrm{E} \chi_{k, \ell^{\prime}} \mathrm{E} \chi_{k, \ell}-\left(\sum_{\ell} \mathrm{E} \chi_{k, \ell}\right)^{2} \\
& =\sum_{\ell}\left(\mathrm{E} \chi_{k, \ell}-\left(\mathrm{E} \chi_{k, \ell}\right)^{2}\right)=N\left(f_{t}\left(y_{k}\right)-f_{t}\left(y_{k}\right)^{2}\right) .
\end{aligned}
$$

Therefore,

$$
\operatorname{Var}\left(\frac{N_{+}(t)-N_{-}(t)}{N}\right)=\frac{1}{N^{2}} \sum_{k} \operatorname{Var}\left(N_{k}(t)\right)=\frac{1}{N} \sum_{k}\left(f_{t}\left(y_{k}\right)-f_{t}\left(y_{k}\right)^{2}\right) \sim \frac{1}{N} \cdot \frac{\sqrt{t}}{\delta x} .
$$

For Case 1, let us assume that the Brownian paths for $k>0$ and $k<0$ are independent. Let

$$
\begin{aligned}
\psi_{\ell}(t, w) & =\sum_{k} \chi_{k, \ell}(t, w) \\
\mathrm{E} \psi_{\ell}^{2} & =\mathrm{E} \sum_{k, k^{\prime}>0} \chi_{k, \ell}(t, w) \chi_{k^{\prime}, \ell}(t, w)+\mathrm{E} \sum_{k, k^{\prime}<0} \chi_{k, \ell}(t, \omega) \chi_{k^{\prime}, \ell}(t, \omega) .
\end{aligned}
$$

Observe that for $0<k^{\prime}<k$,

$$
\chi_{k, \ell}(t, w) \chi_{k^{\prime}, \ell}(t, w)=\chi_{k, \ell}(t, w) .
$$

Similarly for $k<k^{\prime}<0$. Hence,

$$
\mathrm{E} \psi_{\ell}^{2}=\sum_{k}|k| \mathrm{E} \chi_{k, \ell}(t, w)=\sum_{k}|k| f_{t}\left(y_{k}\right) .
$$

Therefore,

$$
\operatorname{Var}\left(\frac{N_{+}(t)-N_{-}(t)}{N}\right)=\frac{1}{N^{2}} \sum_{\ell} \operatorname{Var}\left(\psi_{\ell}\right)=\frac{1}{N} \cdot\left\{\sum_{k}\left(|k| f_{t}\left(y_{k}\right)\right)-\left(\sum_{k} f_{t}\left(y_{k}\right)\right)^{2}\right\} .
$$

As expected this is larger than the variance for Case 2.

The above calculation is based on a piecewise constant reconstruction. We next consider the case of piecewise linear reconstruction: $U(x)=U_{0}+\frac{U_{+}-U_{-}}{\Delta x} x$, where $U_{0}=\frac{U_{+}+U_{-}}{2} . y_{k}$ is now defined by the equation 


$$
y_{k}=\frac{k \delta x}{U_{0}+\frac{U_{+}-U_{-}}{\Delta x} y_{k}} \text {. }
$$

Let $y(x)$ satisfy the relation

$$
y(x) U(y(x))=x
$$

then $d x=U_{0} d y+2 \frac{U_{+}-U_{-}}{\Delta x} y d y$. In the same way as before, we have

$$
\begin{aligned}
\mathrm{E} \frac{N_{+}(t)}{N} & =\sum_{k \geq 0} f_{t}\left(y_{k}\right)=\frac{1}{\delta x} \int_{0}^{\Delta x} f_{t}(y(x)) d x \\
& =\frac{1}{\delta x} \int_{0}^{y(\Delta x)} f_{t}(y)\left(U_{0} d y+2 \frac{U_{+}-U_{-}}{\Delta x} y d y\right) .
\end{aligned}
$$

Let $\Delta y=y(\Delta x)$, then

$$
\begin{aligned}
J(t) & =\frac{\mathrm{E}\left(N_{+}(t)-N_{-}(t)\right)}{N}=\frac{4}{\delta x} \frac{U_{+}-U_{-}}{\Delta x} \int_{0}^{\Delta y} y f_{t}(y) d y \\
& =\frac{4}{\delta x} \frac{U_{+}-U_{-}}{\Delta x}\left\{t G\left(\frac{\Delta y}{\sqrt{2 t}}\right)+\frac{(\Delta y)^{2}}{2} \operatorname{erf}\left(\frac{\Delta y}{\sqrt{2 t}}\right)\right\}
\end{aligned}
$$

where $G(y)=\frac{1}{\sqrt{2 \pi}} \int_{0}^{y} \lambda^{2} e^{-\frac{\lambda^{2}}{2}} d \lambda$. Let $G_{0}=G(+\infty)$. Then

$$
G(y)=G_{0}+O\left(y e^{-\frac{y^{2}}{2}}\right) .
$$

Hence, the time averaged flux behaves as

$$
\frac{J(t)}{t}=C_{0} \frac{U_{+}-U_{-}}{\Delta x}\left(1+O\left(e^{-\frac{(\Delta y)^{2}}{4 t}}\right)\right)
$$

with $C_{0}=\frac{16}{\sqrt{2 \pi}} \frac{G_{0}}{\delta x}$.

This calculation clearly shows the superiority of the piecewise linear reconstruction, in which case the averaged flux rate quickly saturates to a stationary value.

In summary, the error due to compression in time depends on the nature and rate of relaxation to local equilibrium, the compression operators used to extract the $F$-estimators, and the reconstruction operator.

5.3. Subtleties of the Flux-Formulation. Next we turn to the error due to compression in space; i.e., the effect of $Q_{x}$. To see the importance of $Q_{x}$, let us consider the the example of the parabolic homogenization problem. For the macroscale scheme, we will pick the finite difference method. We will take the macroscale flux to be

$$
J_{j+\frac{1}{2}}^{n}=a\left(x, \frac{x}{\varepsilon}\right) \nabla u^{\varepsilon}\left(x_{j+\frac{1}{2}}, t^{n+1}\right) .
$$

From homogenization theory, we know that $u^{\varepsilon}$ has the form

$$
u^{\varepsilon}(x, t)=u_{0}(x, t)+\varepsilon u_{1}\left(x, \frac{x}{\varepsilon}, t\right)+\varepsilon^{2} u_{2}\left(x, \frac{x}{\varepsilon}, t\right)+\ldots
$$


where $u_{0}$ satisfies the homogenized equation

$$
u_{0 t}=\nabla \cdot\left(A(x) \nabla u_{0}\right) .
$$

$A$ is the homogenized coefficient tensor defined earlier, and $u_{1}$ is given by

$$
u_{1}(x, y, t)=\sum_{j} \chi_{j}(x, y) \frac{\partial u_{0}}{\partial x_{j}}(x, t)
$$

where $\chi_{j}$ are the solution of the cell problem

$$
\nabla_{y} \cdot\left(a(x, y)\left(\nabla_{y} \chi_{j}+e_{j}\right)\right)=0
$$

We also know that

$$
\left|\nabla \cdot\left(a\left(x, \frac{x}{\varepsilon}\right) \nabla u^{\varepsilon}\right)-\nabla \cdot\left(A(x) \nabla u_{0}\right)\right|=O(\varepsilon)
$$

as $\varepsilon \rightarrow 0$. However, except in one dimension, it is generally not true that

$$
J^{\varepsilon}(x, t)-\bar{J}(x, t)=a\left(x, \frac{x}{\varepsilon}\right) \nabla u^{\varepsilon}(x, t)-A(x) \nabla u_{0}(x, t) \rightarrow 0 .
$$

Instead, we have

$$
J^{\varepsilon}(x, t)-\bar{J}(x, t)=f_{0}\left(x, \frac{x}{\varepsilon}, t\right)+\varepsilon f_{1}\left(x, \frac{x}{\varepsilon}, t\right)+\ldots
$$

where $f_{0}(x, y, t)$ satisfies $\nabla_{y} \cdot f_{0}=0$. A specific example for which $f_{0}(x, y, t) \neq 0$ will be given below.

The flux form of HMM is able to compute $J^{\varepsilon}(x, t)$ accurately. But notice that

$$
\nabla_{h} \cdot J^{\varepsilon}(x, t)-\nabla_{h} \cdot \bar{J}(x, t)=\nabla_{h} \cdot f_{0}\left(x, \frac{x}{\varepsilon}, t\right)+\varepsilon \nabla_{h} \cdot f_{1}\left(x, \frac{x}{\varepsilon}, t\right)+\ldots
$$

where $\nabla_{h}$. is the discrete divergence operator on the macroscopic grid. The evaluation of $U^{n+1}$ will suffer from the large errors coming from the poor approximation of $\nabla_{h}$. $f_{0}\left(x, \frac{x}{\varepsilon}, t\right)$ to $\nabla \cdot f_{0}\left(x, \frac{x}{\varepsilon}, t\right)$. This problem does not occur if we approximate directly the force $\nabla \cdot\left(A(x) \nabla u_{0}\right)$ since the oscillatory component of $f_{0}$ does not contribute to the force by virtue of $\nabla_{y} \cdot f_{0}(x, y, t)=0$.

This additional error is due to the oscillatory nature of the microscopic fluxes from which macroscopic fluxes are extracted. It is similar in nature to the case of weak relaxation to local equilibrium when error due to compression in time was considered. The solution to this problem is simple. Instead of using pointwise values of the flux at cell boundaries, we have to take appropriate spatial averages to smooth out the spatial oscillations (see [6]).

We now give an example for which $f_{0} \neq 0$. First of all, let us observe that if $f_{0}=0$, then we have

$$
a(x, y)\left(I+\nabla_{y} \chi\right)=A(x)
$$

Hence,

$$
\begin{aligned}
& \nabla_{y} \chi=a(x, y)^{-1} A(x)-I \\
& A(x)=\left(\int_{I} a(x, y)^{-1} d y\right)^{-1} .
\end{aligned}
$$

This holds in one dimension. 
Let us consider a situation when

$$
a(x, y)=\left(\begin{array}{cc}
a\left(y_{1}\right) & 0 \\
0 & a\left(y_{1}\right)
\end{array}\right)
$$

where $a(y)>0$ is a smooth periodic function with period 1 .

It is an easy computation to get the solutions for the cell problem

$$
\begin{aligned}
& \frac{d \chi_{1}}{d y_{1}}=\frac{1}{a\left(y_{1}\right)}\left(\int_{0}^{1} \frac{1}{a\left(y_{1}\right)} d y_{1}\right)^{-1}-1 \\
& \frac{d \chi_{2}}{d y_{1}}=0
\end{aligned}
$$

$\chi_{1}=\chi_{1}\left(y_{1}\right), \chi_{2}=\chi_{2}\left(y_{1}\right)$. The leading order flux is then

$$
J^{\varepsilon}(x, t)=J_{0}\left(x, \frac{x}{\varepsilon}, t\right)
$$

where

$$
\begin{aligned}
J_{0}(x, y, t) & =a(x, y)\left(I+\nabla_{y} \chi(x, y)\right) \nabla_{x} u_{0} \\
& =\left(\begin{array}{c}
\left(\int_{0}^{1} \frac{1}{a(y)} d y\right)^{-1} \partial_{x_{1}} u_{0}(x, t) \\
a\left(y_{1}\right) \partial_{x_{2}} u_{0}(x)
\end{array}\right) .
\end{aligned}
$$

Hence,

$$
f_{0}(x, y, t)=\left(\begin{array}{l}
0 \\
\left(a\left(y_{1}\right)-\int_{0}^{1} a(y) d y\right) \partial_{x_{2}} u_{0}(x, t)
\end{array}\right)
$$

5.4. Limitations of HMM. To appreciate the limitations of HMM, we go back to the example of Carleman equations discussed in Section 2. From the homogenized form of the Carleman equations, (2.35), we see that some essential knowledge of the oscillations must be kept after compression. The nonlinear terms in (2.35) imply that the local distribution of $u$ and $v$ values in the oscillation influence the weak limit via the terms,

$$
\int_{0}^{1} \tilde{v}^{2} d y, \quad \int_{0}^{1} \tilde{u}^{2} d y
$$

and the evolution of these distributions decay along the characteristics from the term $-\tilde{u}^{2}$ and $-\tilde{v}^{2}$.

A local microscale computation, as in the linear homogenization problems discussed earlier, followed by compression to approximate the weak limit will thus not work.

The homogenized form of the equation (2.35) can be used as a basis for HMM, replacing a direct discretization of the original problem (2.34). A discretization in the $y$ fiber is needed but the computational complexity of the algorithm will not increase with decreasing $\varepsilon$.

In this case it is possible, however, to construct an HMM with the same complexity as a coarse grid calculation in $x, t$-space. By choosing appropriate coarse grid discretization a coarse grid sampling of $u, v$ is enough. The details are given in [30]. 
The original compression is thus a uniform sampling with $\Delta x / \varepsilon \neq$ rational. For the initial values we have,

$$
\begin{aligned}
& \left\{U_{i}^{0}\right\}=\left\{a\left(x_{i}, x_{i} / \varepsilon\right)\right\}=Q a(x, x / \varepsilon) \\
& \left\{V_{i}^{0}\right\}=\left\{b\left(x_{i}, x_{i} / \varepsilon\right)\right\}=Q b(x, x / \varepsilon) .
\end{aligned}
$$

\section{Variations and Improvements of the Basic HMM}

The HMM as we have described it requires the definition of $Q$ and thus the macro scale variable $U$. In most of our examples, the choice of coarse-scale variable were quite natural. This may not always be the case, and it is possible to determine $Q$ automatically as part of the computational process. Let us mention two such techniques.

The first is the whole class of model reduction methods that, in particular, are used in connection to control. The dimension of the state space is reduced when the reduced model is derived. The numerical techniques involved may, for example, be singular value decomposition or Krylov subspace methods, [10]. The reduced state space will in our case typically correspond to the macro scale.

The other technique is based on wavelet compression. Such compression is common in signal and image processing but has also been applied to differential equations. In [31], Engquist and Runborg describe a wavelet-based method for numerical homogenization. A differential equation representing both macro- and microscale processes is first discretized on the microscale by standard techniques. The resulting numerical operator is then projected onto a coarse wavelet space. The corresponding compression also reduces the numerical state space and can be used as our $Q$. Examples are given from classical homogenization for which the compressed dependent variable corresponds to the standard macroscale.

The numerical methods for the coarse grid that we have presented have mainly been of low order. This is advantageous in the presentation of the principles but in practical computation it is often better to apply higher order methods. This fits well into our general framework. Higher order elements can replace $P_{1}$ in the variational problems and higher order differencing, and reconstruction are possible in the Godunov setting.

In order to apply these higher order methods, higher order of fluxes or forces from the microscale simulation is required. This is achieved by appropriate choice of $F$-estimators.

Assume that $f(x)$ is the microscale force computed from a microscale simulation for $x \in \Omega_{\delta} \subset \mathbb{R}^{d}$, where $\Omega_{\delta}$ is a computational cell in mcd, with diameter $\left(\Omega_{\delta}\right)=\delta$. $x$ can represent both spatial and temporal variables. Assume also that $f(x)$ has the form:

$$
f(x)=F(x)+b(x, \varepsilon)
$$

where $F$ is the macroscale forcing that we would like to approximate. We would like to estimate $F$ from $f$ using

$$
F(\bar{x})=\int_{\Omega_{\delta}} K(x, \bar{x}) f(x) d x
$$

where $K$ is defined such that

$$
\int_{\Omega_{\delta}} K(x, \bar{x})(x-\bar{x})^{p} d x=\left\{\begin{array}{l}
1, p=0 \\
0,1 \leq|p| \leq p .
\end{array}\right.
$$


When $\bar{x} \in \Omega_{\delta}$ and $f$ is smooth then $F=f$ and $K(x, \bar{x})$ can be chosen as $\delta(x-\bar{x})$. If $\Omega_{\delta}$ contains a subdomain $\Omega_{\delta}^{\prime}$ in which $f$ has a transient, $K$ should be supported outside of $\Omega_{\delta}^{\prime}$. In the case when $f$ contains oscillations, then $K$ should be smooth as a function of $x$ and compactly supported in $\Omega_{\delta} \backslash \Omega_{\delta}^{\prime}$.

To be more specific, if $f$ is highly oscillatory around a smooth mean function $F(x)$, then we can write $b$ as

$$
\frac{\partial B}{\partial x_{k}}=b_{k}(x, \varepsilon),|B(x, \varepsilon)| \leq C \varepsilon, 1 \leq k \leq d .
$$

Hence,

$$
\begin{aligned}
\int_{\Omega_{\delta}} K(x, \bar{x}) f(x) d x & =\int_{\Omega_{\delta}} K(x, \bar{x})\left(\sum_{|j| \leq p} F_{j}(x-\bar{x})^{j}\right) d x+O\left(\delta^{p+1}\right)+\int_{\Omega_{\delta}} K(x, \bar{x}) b(x, \varepsilon) d x \\
& =F(\bar{x})+O\left(\delta^{p+1}\right)+\int_{\Omega_{\delta}} \frac{\partial K(x, \bar{x})}{\partial x_{k}} B(x, \varepsilon) d x \\
& =F(\bar{x})+O\left(\delta^{p+1}+\varepsilon \delta^{-1}\right) .
\end{aligned}
$$

The final step for the high order numerical $F$-estimator is replacing the integral in (6.2) by a high order, accurate quadrature formula.

In this paper, we concentrated on the case when the missing macroscale data depend only on local behavior of the macroscale quantities. This is not true for many practical problems. The incompressible limit of fluid flows is a good example. There the missing macroscale data, the pressure, depends on the velocity field in a nonlocal way. It is possible to modify the HMM procedure presented here to accommodate such situations. We will postpone a detailed discussion of this extension to a later publication [23].

Some knowledge about the macroscopic model is necessary in order to guarantee consistency with the macroscopic problem. For example it is important to know whether the macroscopic model is nonlocal, and if it is local, it is helpful to know the order of the differential equations. Such information might be probed in a preprocessing step or adaptively during the computation using the microscopic model. This too will be considered in more detail in a future publication [23].

For Type A problems, it often happens that near the defects or singularities, $t_{R}$ is no longer small compared with $t_{M}$. In such cases, it is important to modify HMM locally so that the microscopic states are retained near defects or singularities, instead of being converted to the macrostates at the end of each macro time step. This is already done in algorithms involving multilevels of physical models that are coupled together locally $[1,2,26,27,65]$.

\section{Conclusion}

The heterogeneous multiscale method (HMM) is a general methodology that allows us to efficiently move between the macroscopic and microscopic models, and to best exploit scale separation in the problem for improving efficiency. As is the case with other fast algorithms, such as multigrid, fast multipole method, and waveletbased multiresolution methods, the efficiency is achieved through minimizing redundancy in the computations. For HMM, this is done through (1) compressing the complexity of the microscopic solvers, (2) using explicit macroscopic models when they are valid and available, and (3) using available computational data. As we 
demonstrated with several examples, this approach offers considerable flexibility.

There are two main components in HMM. The first is to formulate the microscopic problem on the smallest possible computational domain without altering its basic local averaged properties. This includes prescribing the right constraints, and processing the data to extract the macroscopic information efficiently and accurately. The second component is information passing at the macroscopic level by linking correctly the different microscopic problems in order to model the macroscopic behavior. This is done in HMM through the overall macroscale scheme. We have given several versions on how this can be implemented.

In order not to be overwhelmed by the generality of HMM, we summarize here some examples of new algorithms that emerge from applications to specific problems.

1. The homogenization problem. For both the static and dynamic homogenization problems (2.6),(4.9), our method proceeds as a standard finite element method with the usual basis functions. The new component comes in the computation of the stiffness matrix which is obtained by solving a small "cell problem" on each element. Additional savings will be obtained if the semi-empirical methods are used (see Figure 4). The flexibility of HMM means that it can be readily applied to more complicated problems such as nonlinear problems or if the microstructure changes with time.

In contrast, the methods proposed in $[7,45]$ modify the basis functions by building in the microstructures. This means that the microstructure problem has to be solved over the whole element. It also limits the applicability of the method to problems for which the microstructures can be found beforehand.

2. Coupling molecular dynamics with thermoelasticity. At low temperatures, there exist satisfactory algorithms that couple molecular dynamics with continuum elasticity. For static and quasistatic problems, the quasicontinuum method is now a popular tool [73, 69, 68]. For dynamic problems, the dynamic atomistic/continuum method proposed in [26, 27] works quite well. These methods are ideal for studying crystalline solids with isolated defects. The natural next step is to deal with the effect of finite temperature and heat conduction. HMM can be readily used for this purpose. Away from defects and interfaces, one uses the continuum theory of thermoelasticity with material parameters obtained from molecular dynamics. Near the defects where the local deformation is large, one uses the compressed microscopic model to evaluate the fluxes or forces in the macroscopic equations. The details of the microscopic expression of the fluxes can be found in Section 2 .

In the same fashion, HMM provides a way of coupling molecular dynamics with continuum theory of fluid flow.

3. Structure and dynamics of complex interfaces. For complex interfaces such as turbulent flames, twin boundaries and grain boundaries for which the interfacial equations are not explicitly known, HMM provides an efficient way of evolving the interfaces using the microscopic models and at the same time coupling them to the macroscopic behavior away from the interfaces.

4. Coupled hydrodynamic/kinetic models for complex fluids [9]. When molecular conformation has a nontrivial contribution to macroscopic stress, HMM provides an efficient way of exploiting the separation between relaxational and hydrodynamic time scales.

There are numerous other possible areas of applications that we will not enumerate here.

From a theoretical point of view, we can associate an underlying macroscopic scheme, the Generalized Godunov Scheme (GGS), to HMM. The stability properties of 
HMM can be read off from the GGS. The error of HMM comes from two main sources: the standard truncation error and the additional error due to compression. The compression error also comes from two sources: compression in time and compression in space. The error due to compression in time depends on the nature and rate of relaxation to local equilibrium, as well as the compression operators used to extract the $F$-estimators. Similarly the error due to compression in space also depends on the compression operators used in the $F$-estimators and the boundary and constraining conditions imposed when truncating the microscopic computational domains.

Acknowledgement. We are grateful for many inspiring discussions with Yannis Kevrekidis in which he has outlined his program of macroscale analysis based on microscale solvers. We are also grateful to Eric Vanden-Eijnden and Olof Runborg for stimulating discussions and to Assyr Abdulle and Chris Schwab for suggestions that improved the first draft of the paper. The work of $\mathrm{E}$ is supported in part by an ONR grant N00014-01-1-0674. The work of Engquist is supported in part by NSF grant DMS-9973341.

\section{REFERENCES}

[1] F.F. Abraham, J.Q. Broughton, N. Bernstein, and E. Kaxiras, Spanning the continuum to quantum length scales in a dynamic simulation of brittle fracture. Europhys. Lett., 44:783$787,1998$.

[2] F.F. Abraham, J.Q. Broughton, N. Bernstein, and E. Kaxiras, Concurrent coupling of length scales: Methodology and application, Phys. Rev. B, 60:2391-2402, 1999.

[3] G. Allaire, Homogenization and two-scale convergence. SIAM J. Math. Anal 23:1482-1518, 1992.

[4] V. Arnold, Mathematical Methods in Classical Mechanics. Springer-Verlag.

[5] A. Abdulle, Fourth order Chebychev methods with recurrence relations. SIAM J. Sci. Comput., to appear.

[6] A. Abdulle and W. E, Finite difference HMM for homogenization problems. submitted to J. Comput. Phys.

[7] I. Babuska, Homogenization and its applications. SYNSPADE 1975, B.Hubbard(ed), 89-116.

[8] A. Benssousan, J.L. Lions, and G. Papanicolaou, Asymptotic Analysis of Periodic Structures. North-Holland, 1978.

[9] R.B. Bird, C.F. Curtiss, R.C. Armstrong, and O. Hassager, Dynamics of Polymeric Liquids, 2: Kinetic Theory. John Wiley, New York, 1987.

[10] D.L. Boley, Krylov space methods on state-space control methods. Circ. Syst. Signal Proc., 13:733-758, 1994

[11] A. Brandt, Multigrid methods in lattice field computations. Nuclear Physics B Proc. Suppl. 26:137-180, 1992

[12] A. Brandt, Multiscale Scientific Computation: Review 2001. Lecture Notes in Computational Science and Engineering, T.J. Barth et al. (eds)., Springer, 2002.

[13] R. Car and M. Parrinello, Unified approach for molecular dynamics and density-functional theory. Phys. Rev. Lett., 55:2471-2474, 1985.

[14] W. Cai, M. de Koning, V.V. Bulatov, and S. Yip, Minimizing boundary reflections in coupleddomain simulations. Phys. Rev. Lett., 85:3213-3216, 2000.

[15] A.J. Chorin, A. Kast, and R. Kupferman, Optimal prediction of underresolved dynamics. Proc. Nat. Acad. Sc. USA, 95:4094-4098, 1998.

[16] A.J. Chorin, O. Hold, and R. Kupferman, Optimal prediction with memory. Physica D, In press.

[17] P.G. Ciarlet, The Finite Element Methods for Elliptic Problems. Amsterdam; New York: North-Holland Pub. Co., 1978.

[18] R. Clayton and B. Engquist, Absorbing boundary conditions for acoustic and elastic wave equations. Bull. Seismol. Soc. Amer., 67:1529-1540, 1977.

[19] C. Curtarolo, Dynamic of a nonhomogeneously coarse-grained system. Phys. Rev. Lett, In press.

[20] S.R. de Groot and P. Mazur, Nonequilibrium Thermodynamics. Dover Publications, Inc., New York, 1984 
[21] E.B. Dussan V, On the spreading of liquids on solid surfaces: Static and dynamic contact lines. Ann. Rev. Fluid Mech., 11:371-400, 1979.

[22] W. E, Homogenization of linear and nonlinear transport equations. Comm. Pure and Appl., XLV:301-326, 1992.

[23] W. E and B. Engquist, In preparation.

[24] W. E and B. Engquist, Heterogeneous multiscale methods for homogenization problems. submitted to Mulitiscale Modeling and Simulation.

[25] W. E, B. Engquist, and C.W. Shu, In preparation.

[26] W. E and Z. Huang, Matching conditions in atomistic-continuum modeling of materials. Phys. Rev. Lett., 87:135501, 2001.

[27] W. E and Z. Huang, A dynamic atomistic-continuum method for the simulation of crystalline materials. J. Comput. Phys., 182:234-261, 2002.

[28] W. E, P.B. Ming, and P. Zhang, Analysis of the heterogeneous multiscale method for homogenization problems. Preprint

[29] W. E, W. Ren, and E. Vanden-Eijnden, Probing multiscale energy landscapes using the string method. submitted to Phys. Rev. Lett.

[30] B. Engquist, Computation of oscillatory solutions to hyperbolic differential equations. Springer Lecture Notes in Mathematics, 1270:10-22, 1987.

[31] B. Engquist and O. Runborg, Wavelet-Based Numerical Homogenization with Applications. Lecture Notes in Computational Science and Engineering, T.J. Barth et al. (eds)., Springer, 2002.

[32] B. Engquist and A. Majda, Radiation boundary conditions for acoustic and elastic wave calculations. Comm. Pure Appl. Math., 32:313-357, 1979.

[33] X.J. Fan, Molecular models and flow calculations: II. Simulation of steady planar flow. Acta Mech. Sinica, 5:216-226, 1989.

[34] C.W. Gear and I.G. Kevrekidis, Projective methods for stiff differential equations: problems with gaps in their eigenvalue spectrum. Submitted to SIAM J. Sci. Comp.

[35] C.W. Gear, I.G. Kevrekidis, and C. Theodoropoulos, 'Coarse' integration/bifurcation analysis via microscpic simulators: Micro-Galerkin methods. Submitted to Comp. Chem. Eng.

[36] G. Giacomin, J.L. Lebowitz, and E. Presutti, Deterministic and stochastic hydrodynamic equations arising from simple microscopic model systems. In Stochastic Partial Differential Equations: Six Perspectives, R. Carmona and B. Rozovskii(eds.), American Mathematical Society, 1999.

[37] J. Glimm, O. McBryan, R. Menikoff, and D.H. Sharp, Front tracking applied to Rayleigh-Taylor instability. SIAM J. Sci. Statist. Comput. 7:230-251, 1986

[38] S.K. Godunov, Mat. Sb., 47:271, 1959.

[39] A. Gulliou and B. Lago, Domaine de stabilité associé aux formules d'intégration numérique d'équations différentielles, à pas séparés et à pas liés. ler Congr. Assoc. Fran. Calcul, AFCAL, Grenoble, 43-56, Sept. 1960.

[40] W. Hackbusch, Convergence of multigrid iterations applied to difference equations. Math. Comp., 34:425-440, 1980

[41] E. Hairer and G. Wanner, Solving Ordinary Differential Equations II, Stiff and DifferentialAlgebraic Problems. Springer-Verlag, 1991.

[42] P. Hänggi, P. Talkner, and M. Borkovec, Reaction-rate theory, fifty years after Kramers. Rev. Modern Phys., 62:251, 1990.

[43] J.A. Harrison and D.W. Brenner, Atomic-Scale Simulation of Tribological and Related Phenomena. In Handbook of Micro/Nano Tribology, B. Bhushan(ed.), CRC Press, 1995.

[44] A. Harten, P. Lax, and B. Van Leer, SIAM Review, 25, 1983.

[45] T. Hou and X. Wu, A multiscale finite element method for elliptic problems in composite materials and porous media. J. Comput. Phys., 134:169-189, 1997.

[46] T.J.R. Hughes, Multiscale phenomena: Green's functions, the Dirichlet to Neumann formulation, subgrid, scale models, bubbles and the origin of stablized methods. Comput. Methods Appl. Mech. Engrg., 127:387-401, 1995.

[47] L. Landau and E.M. Lifshitz, Statistical Physics. Part 1, 3rd edition, Butterworth-Heinemann, 1980.

[48] M. Laso and H.C. Ottinger, Calculation of viscoelastic flow using molecular models: The CONNFFESSIT approach. J. Non-Newtonian Fluid Mech., 47:1-20, 1993

[49] V.I. Lebedev and S.I. Finogenov, Explicit methods of second order for the solution of stiff systems of ordinary differential equations. Zh. Vychisl. Mat. Mat Fiziki, 16:895-910, 1976.

[50] C.D. Levermore and W.J. Morokoff, The Gaussian moment closure for gas dynamics. SIAM J. Appl. Math., 59:72-96(electronic), 1999.

[51] R. LeVeque, Numerical Methods for Conservation Laws, Birkhäuser, 1990. 
[52] M. Marder and S. Gross, Origin of crack tip instabilities. J. Mech. Phys. Solids, 43:1-48, 1995.

[53] A.G. Markeev, D. Maroudas, and I.G. Kevrekidis, 'Coarse' stability and bifurcation analysis using stochastic simulators: Kinetic Monte Carlo Examples. Submitted to J. Chem. Phys.

[54] R. Miller, E.B. Tadmor, R. Phillips, et al, Quasicontinuum simulation of fracture at the atomic scale. Model Simul. Mater. Sc., 6:607-638, 1998.

[55] P. Moin, Numerical Issues in Large Eddy Simulation of Complex Turbulent Flows and Application to Aeroacoustics. Advanced Turbulent Flow Computations (Udine, 1998), 131-154, CISM Courses and Lectures, 395, Springer, Vienna, 2000.

[56] G. Nguetseng, A general convergence result for a functional related to the theory of homogenization. SIAM J. Math. Anal. 20:608-623, 1989.

[57] S. Osher and J. Sethian, Front propagation with curvature-dependent speed: Algorithms based Hamilton-Jacobi formulations. J. Comput. Phys., 112:334-363, 1994.

[58] C. Peskin and D.M. McQueen, Computational biofluid dynamics. Fluid Dynamics in Biology (Seattle, WA, 1991), 161-186, Contemp. Math., 141, Amer. Math. Soc., Providence, RI, 1993.

[59] N. Peters, Turbulent Combustion. Cambridge University Press, Cambridge, 2000.

[60] R. Phillips, Crystals, Defects and Microstructures. Cambridge University Press, 2001.

[61] M.O. Robbins and M.H. Müser, Computer Simulations of Friction, Lubrication and Wear. in Modern Tribology Handbook, B. Bhushan(ed.), CRC Press, 2001.

[62] R.E. Rudd and J.Q. Broughton, Coarse-grained molecular dynamics and the atomic limit of finite elements. Phys. Rev. B, 58:R5893-R5896, 1998.

[63] R.E. Rudd and J.Q. Broughton, Atomistic simulation of MEMS resonators through the coupling of length scales. J. Modeling and Simulation of Microsystems, 1:29-38, 1999.

[64] O. Runborg, C. Theodoropoulos, and I.G. Kevrekidis, Effective bifurcation analysis: Timestepper based approach. Submitted to Nonlinearity.

[65] T. Schulze, P. Smereka, and W. E, Coupling kinetic Monte-Carlo with continuum models with applications to epitaxial growth. submitted to J. Comput. Phys.

[66] C. Schwab and A.-M. Matache, Two-Scale FEM for Homogenization Problems. Proceedings of the Conference "Mathematical Modelling and Numerical Simulation in Continuum Mechanics," Yamaguchi, Japan, I. Babuska, P.G. Ciarlet, and T. Myoshi (eds.), Lecture Notes in Computational Science and Engineering, Springer Verlag, 2002.

[67] C. Schwab and A.-M. Matache, Generalzied FEM for Homogenization Problems. Lecture Notes in Computational Science and Engineering, T.J. Barth et al. (eds), Springer, 2002.

[68] V.B. Shenoy, R. Miller, and E.B. Tadmor, et al, Quasicontinuum models of interfacial structure and deformation. Phys. Rev. Lett., 80:742-745, 1998.

[69] V.B. Shenoy, R. Miller, E.B. Tadmor, D. Rodney, R. Phillips, and M. Ortiz, An adaptive finite element approach to atomic-scale mechanics-the quasicontinuum method. J. Mech. Phys. Solids, 47:611-642, 1999.

[70] G.S. Smith, E.B. Tadmor, and E. Kaxiras, Multiscale simulation of loading and electrical resistance in silicon nanoindentation. Phys. Rev. Lett., 84:1260-1263, 2000.

[71] H. Spohn, Large Scale Dynamics of Interacting Particles. Springer-Verlag, 1991.

[72] G. Strang, Accurate partial differential methods II: Nonlinear problems. Numer. Math., 6:3746,1964 .

[73] E.B. Tadmor, M. Ortiz, and R. Phillips, Quasicontinuum analysis of defects in crystals. Phil. Mag., A73:1529-1563, 1996.

[74] L. Tartar, Solutions oscillantes des quations de Carleman. Goulaouic-Meyer-Schwartz Seminar, 1980-1981, Exp. No. XII, 15 cole Polytech., Palaiseau, 1981.

[75] C. Theodoropoulos, Y.-H. Qian, and I.G. Kevrekidis, 'Coarse' stability and bifurcation analysis using time-steppers: A reaction-diffusion example. PNAS, 97:9840-9843, 2000.

[76] A.-K. Tornberg and B. Engquist, Interface tracking in two-phase flows. Multifield Problems, State of the Art, Springer Verlag, 58-66, 2000.

[77] S. Torquato, Random Heterogeneous Materials: Microstructure and Macroscopic Properties. Springer-Verlag, 2001.

[78] E. Vanden-Eijnden, In preparation.

[79] K. Xu and K.H. Prendergast, Numerical Navier-Stokes solutions from gas kinetic theory. J. Comput. Phys., 114:9-17, 1994.

[80] J. Suen, Y.L. Joo, and R.C. Armstrong, Molecular Orientation Effects in Viscoelasticity. Annual Review of Fluid Mechanics, 2002.

[81] H.T. Yau, Asymptotic Solutions to Dynamics of Many-Body Systems and Classical Continuum Equations. In Current Development in Mathematics, International Press, 2000. 